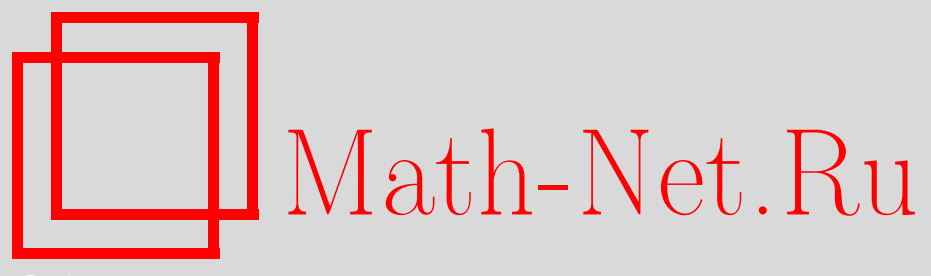

А. С. Холево, Гауссовские оптимизаторы и проблема аддитивности в квантовой теории информации, УМН, 2015, том 70, выпуск 2, 141-180

DOI: https://doi.org/10.4213/rm9634

Использование Общероссийского математического портала Math-Net.Ru подразумевает, что вы прочитали и согласны с пользовательским соглашением http://www . mathnet.ru/rus/agreement

Параметры загрузки:

IP : 54.81 .137 .203

26 апреля 2023 г., 17:12:02

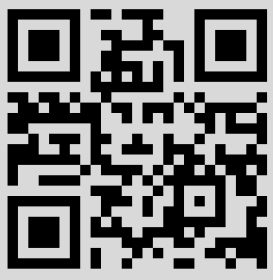




\title{
Гауссовские оптимизаторы и проблема аддитивности в квантовой теории информации
}

\author{
А. С. Холево
}

Дается обзор двух замечательных аналитических проблем квантовой теории информации. Основную часть составляет подробное изложение недавнего (частичного) решения квантовой проблемы гауссовских оптимизаторов, которое устанавливает оптимальное свойство глауберовских когерентных состояний - специального случая чистых квантовых гауссовских состояний. Мы развиваем понятие квантового гауссовского канала как некоммутативного аналога интегрального оператора с гауссовским ядром и доказываем, что когерентные состояния, и при определенных условиях только они, минимизируют широкий класс вогнутых функционалов от выходного состояния гауссовского канала. Таким образом, выходные состояния, соответствующие гауссовскому входу, являются "наименее хаотичными", мажорируя все другие выходные состояния. Решение, однако, существенно ограничено калибровочно-инвариантным случаем, в котором особую роль играет выделенная комплексная структура.

Мы также обсуждаем известную гипотезу аддитивности, которая была в принципе решена в отрицательном смысле около пяти лет назад. Эта гипотеза относится к свойствам аддитивности или мультипликативности (относительно тензорных произведений каналов) информационных величин, связанных с классической пропускной способностью квантового канала, таких как $(1 \rightarrow p)$-нормы канала или минимальные выходные энтропии фон Неймана и Реньи. Замечательное следствие настоящего решения квантовой проблемы гауссовских оптимизаторов заключается в том, что эти свойства аддитивности, не справедливые в общем случае, выполняются в важном и интересном классе калибровочно-ковариантных гауссовских каналов.

Библиография: 65 названий.

Ключевые слова: вполне положительное отображение, канонические коммутационные соотношения, гауссовское состояние, когерентное состояние, квантовый гауссовский канал, калибровочная ковариантность, энтропия фон Неймана, пропускная способность, мажоризация.

DOI: $10.4213 / \mathrm{rm} 9634$

Исследование выполнено за счет гранта Российского научного фонда (проект 14-21-00162). 


\section{СоДЕРЖАНИЕ}

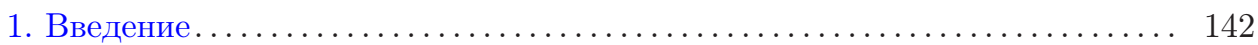

2. Проблема аддитивности для квантовых каналов................. 144

2.1. Определение канала............................. 144

2.2. Представление типа Стайнспринга................... 146

2.3. Энтропийные количества и аддитивность................. 148

2.4. Пропускная способность канала ....................... 149

2.5. Основные выводы . . . . . . . . . . . . . . . . . . . . 150

2.6. Мажоризация для квантовых состояний................. 152

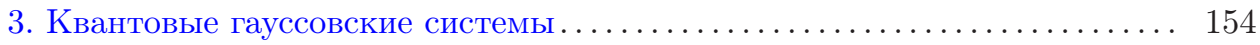

3.1. Гауссовские состояния и каналы ..................... 154

3.2. Комплексные структуры и калибровочная симметрия.......... 157

3.3. Аттенюаторы и усилители . ......................... 160

3.4. Гауссовские оптимизаторы .......................... 165

3.5. Явные формулы и аддитивность .................... 168

3.6. Случай квантово-классического гауссовского канала ......... 173

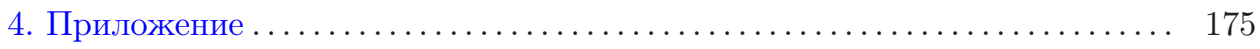

Список литературы .................................... 177

\section{1. Введение}

Квантовая проблема гауссовских оптимизаторов - это аналитическая задача, которая возникла в квантовой теории информации в конце прошлого века и которая представляет самостоятельный математический интерес. Лишь недавно было найдено ее решение [22], [53] в достаточно общей ситуации, в то время как в полном объеме проблема по-прежнему остается открытой. Чтобы объяснить ее природу и возникающие трудности, мы начнем с родственной классической проблемы гауссовских максимизаторов, которая получила достаточно исчерпывающее решение (см. работу Либа [51] и цитированную в ней литературу). Рассмотрим интегральный оператор $G$ из $L_{p}\left(\mathbb{R}^{s}\right)$ в $L_{q}\left(\mathbb{R}^{r}\right)$, заданный гауссовским ядром (т. е. экспонентой от квадратичной формы) с $(q \rightarrow p)$-нормой

$$
\|G\|_{q \rightarrow p}=\sup _{f \neq 0} \frac{\|G f\|_{p}}{\|f\|_{q}}=\sup _{\|f\|_{q} \leqslant 1}\|G f\|_{p} .
$$

При определенных достаточно широких предположениях относительно квадратичной формы, определяющей ядро, а также относительно $p$ и $q$ такой оператор корректно определен и супремум в (1) достигается на гауссовской функции $f$. Более того, при некоторых дополнительных ограничениях всякий максимизатор является гауссовским. Как гласит заголовок статьи [51], "гауссовские ядра имеют только гауссовские максимизаторы".

Знание того, что максимизатор гауссов, может быть использовано для вычисления точного значения нормы (1); это было отправной точкой классических результатов Бабенко [5] и последующей работы Бекнера [6], в которой 
были найдены наилучшие константы для неравенства Хаусдорфа-Юнга, относящегося к $\left(p \rightarrow p^{\prime}\right)$-норме $\left(p^{-1}+\left(p^{\prime}\right)^{-1}=1,1<p \leqslant 2\right)$ преобразования Фурье (которое, очевидно, задается вырожденным мнимым гауссовским ядром).

Трудность задачи оптимизации (1) состоит в том, что она требует максимизации выпуклой функции, поэтому в данном случае общая теория выпуклой оптимизации не слишком полезна (из нее лишь следует, что максимизатор нормы $\|G f\|_{p}$ принадлежит грани выпуклого множества $\left.\|f\|_{q} \leqslant 1\right)$. Решение задачи основано на существенном использовании классического неравенства Минковского и вытекающей из него мультипликативности классических $(q \rightarrow p)$-норм относительно тензорных произведений интегральных операторов.

Интересным применением этих классических результатов в квантовой математической физике явилось данное Либом доказательство [50] гипотезы Верля [63]. Пусть $\rho$ - оператор плотности в сепарабельном гильбертовом пространстве $\mathscr{H}$, представляющий состояние квантовой системы; "классическая энтропия" состояния $\rho$ определяется как ${ }^{1}$

$$
H_{\mathrm{cl}}(\rho)=-\int_{\mathbb{C}} p_{\rho}(z) \log p_{\rho}(z) \frac{d^{2} z}{\pi},
$$

где $p_{\rho}(z)=\langle z|\rho| z\rangle$ - диагональное значение ядра оператора $\rho$ в системе глауберовских когерентных векторов ${ }^{2}\{|z\rangle ; z \in \mathbb{C}\}$ [48], [33]. Гипотеза состояла в том, что $H_{\mathrm{cl}}(\rho)$ принимает минимальное значение, когда $\rho$ само является когерентным состоянием, т. е. проектором на один из когерентных векторов. Либ [50] использовал точные значения констант в неравенстве Хаусдорфа-Юнга для $L_{p}$-норм преобразования Фурье [5], [6] и в неравенстве Юнга для свертки [6], чтобы доказать соответствующую гипотезу о максимизаторах в случае $f(x)=x^{p}$, и рассмотрел предел $\lim _{p \downarrow 1}(1-p)^{-1}\left(1-x^{p}\right)=-x \log x$.

Недавно Либ и Соловей [52], используя совершенно иной подход, основанный на изучении спиновых когерентных состояний, усилили результат работы [50]: они показали, что глауберовские когерентные состояния минимизируют любой функционал вида $\int_{\mathbb{C}} f(\langle z|\rho| z\rangle) \frac{d^{2} z}{\pi}$, где $f(x), x \in[0,1],-$ неотрицательная вогнутая функция такая, что $f(0)=0$.

В терминологии квантовой теории информации аффинное отображение $G: \rho \rightarrow p_{\rho}(z)$, переводящее операторы плотности $\rho$ (квантовые состояния) в плотности распределений вероятностей $p_{\rho}(z)$ (классические состояния), является "квантово-классическим каналом" [38]. Более того, оно переводит гауссовские операторы плотности $\rho$ (см. определение в п. 3.1) в плотности гауссовских распределений и в этом смысле является "гауссовским каналом". С этой точки зрения классическая энтропия Верля $H_{\mathrm{cl}}(\rho)$ является выходной энтропией данного канала, и результат Либа означает, что она минимизируется чистым гауссовским состоянием $\rho$. Более того, соответствующий результат для $f(x)=x^{p}$ может быть интерпретирован как утверждение о "гауссовском максимизаторе"

\footnotetext{
${ }^{1}$ Всюду далее основание логарифмов - фиксированное число $a>1$. В теории информации естествен выбор $a=2$, тогда все энтропийные количества измеряются в "битах".

${ }^{2}$ В анализе им соответствуют комплексно-параметризованные гауссовские "всплески". Отметим, что это единственное место в статье, где мы формально воспользовались обозначениями Дирака, непривычными для математика.
} 
для нормы $\|G\|_{1 \rightarrow p}$. Отметим, что значение $q=1$, которое обычно исключается в классической проблеме, в квантовом (некоммутативном) случае играет основную роль.

Рассматриваемая в настоящей работе квантовая проблема гауссовских оптимизаторов относится к квантовым гауссовским каналам, которые являются некоммутативным аналогом гауссовских марковских ядер, и, аналогичным образом, состоит в максимизации выпуклых функций (или минимизации вогнутых, таких как энтропия) от выходных состояний канала, тогда как аргументом является входное состояние. Общая гипотеза состоит в том, что оптимизатор находится в классе чистых гауссовских состояний. Проблема, сформулированная в [42] в контексте квантовой теории информации, несмотря на естественность формулировки, не поддавалась многочисленным попыткам решения в течение ряда лет. Здесь следует отметить точное вычисление классической пропускной способности "канала с чистыми потерями" [21] и доказательство аддитивности энтропий Реньи целого порядка $p$ [24] для специальных моделей каналов. Даже при ограничении класса входных состояний гауссовскими проблема оптимизации остается нетривиальной [56], [31]. Имелась некоторая надежда, что в решении этой проблемы, как и в доказательстве гипотезы Верля, могли бы помочь классические результаты о "гауссовских максимизаторах". Однако решение, найденное недавно в работах Джованнетти, Холево, Гарсия-Патрона [22] и Мари, Джованнетти, Холево [53], использует совершенно иные идеи, основанные на тщательном исследовании структурных свойств квантовых гауссовских каналов. Как уже упоминалось, решение классической проблемы использует неравенство Минковского и вытекающую из него мультипликативность $(q \rightarrow p)$-норм. Однако известный некоммутативный аналог неравенства Минковского [12] не гарантирует мультипликативность норм (или аддитивность соответствующих энтропийных количеств). Более того, недавно было показано, что проблема аддитивности в квантовой теории информации [37] имеет, вообще говоря, отрицательное решение [26]. Мы показываем, что, замечательным образом, решение квантовой проблемы гауссовских оптимизаторов, полученное в [22], влечет также и доказательство свойства мультипликативности/аддитивности для ограниченного класса калибровочно-ковариантных или контравариантных квантовых гауссовских каналов.

Было бы весьма интересно исследовать возможность применения такого подхода для получения некоммутативных обобщений классических результатов о "гауссовских максимизаторах" для $(q \rightarrow p)$-норм. Такого рода обобщения могли бы пролить новый свет на проблему гиперконтрактивности для квантовых динамических полугрупп и некоммутативные аналоги логарифмических неравенств Соболева (см., например, [62]).

\section{2. Проблема аддитивности для квантовых каналов}

2.1. Определение канала. Пусть $\mathscr{H}$ - сепарабельное комплексное гильбертово пространство, $\mathfrak{L}(\mathscr{H})$ - алгебра всех ограниченных операторов в $\mathscr{H}$ и $\mathfrak{T}(\mathscr{H})$ - идеал ядерных операторов. Пространство $\mathfrak{T}(\mathscr{H})$, снабженное следовой нормой $\|\cdot\|_{1}$, является банаховым пространством, которое полезно рассматривать как некоммутативный аналог пространства $L_{1}$. Выпуклое подмно- 
жество пространства $\mathfrak{T}(\mathscr{H})$

$$
\mathfrak{S}(\mathscr{H})=\left\{\rho: \rho^{*}=\rho \geqslant 0, \operatorname{Tr} \rho=1\right\}
$$

является базой положительного конуса в $\mathfrak{T}(\mathscr{H})$. Операторы $\rho$ из $\mathfrak{S}(\mathscr{H})$ называются операторами плотности или квантовыми состояниями. Крайние точки этого пространства состояний образуют подмножество

$$
\mathfrak{P}(\mathscr{H})=\left\{\rho: \rho \geqslant 0, \operatorname{Tr} \rho=1, \rho^{2}=\rho\right\} .
$$

Таким образом, крайние точки множества $\mathfrak{S}(\mathscr{H})$, называемые чистыми состояниями, являются одномерными проекторами $\rho=P_{\psi}$, где $\psi \in \mathscr{H}$ - единичный вектор (см., например, [55]).

Нас будет интересовать класс отображений, которые являются некоммутативным аналогом марковских отображений (линейных, положительных, нормированных отображений) в классическом анализе и теории вероятностей. Пусть $\mathscr{H}_{A}, \mathscr{H}_{B}$ - два гильбертова пространства, которые будут называться входным и выходным пространствами соответственно. Отображение $\Phi: \mathfrak{T}\left(\mathscr{H}_{A}\right) \rightarrow \mathfrak{T}\left(\mathscr{H}_{B}\right)$ называется положительным, если неравенство $X \geqslant 0$ влечет за собой неравенство $\Phi[X] \geqslant 0$, и вполне положительным [61], [54], если отображения $\Phi \otimes \operatorname{Id}_{(d)}$ положительны для всех $d=1,2, \ldots$, где $\operatorname{Id}_{(d)}$ - тождественное отображение алгебры $\mathfrak{L}_{d}=\mathfrak{L}\left(\mathbb{C}^{d}\right)$ всех комплексных $(d \times d)$-матриц. Равносильно, для любой неотрицательно определенной блочной матрицы $\left[X_{j k}\right]_{j, k=1, \ldots, d}$ матрица $\left[\Phi\left[X_{j k}\right]\right]_{j, k=1, \ldots, d}$ является неотрицательно определенной.

Линейное отображение $\Phi$ сохраняет след, если $\operatorname{Tr} \Phi[X]=\operatorname{Tr} X$ для всех $X \in \mathfrak{T}\left(\mathscr{H}_{A}\right)$.

ОПРЕДЕЛЕНИЕ 1. Квантовым каналом называется линейное, вполне положительное, сохраняющее след отображение $\Phi: \mathfrak{T}\left(\mathscr{H}_{A}\right) \rightarrow \mathfrak{T}\left(\mathscr{H}_{B}\right)$. Символ $A$ будет ассоциироваться со входом канала, а символ $B-$ с выходом. Иногда для сокращения обозначений мы будем писать просто $\Phi: A \rightarrow B$.

Очевидно, что всякий канал является положительным отображением, переводящим состояния в состояния: $\Phi\left[\mathfrak{S}\left(\mathscr{H}_{A}\right)\right] \subseteq \mathfrak{S}\left(\mathscr{H}_{B}\right)$. Поскольку $\mathfrak{T}(\mathscr{H})-$ пространство, нормированное базой, отсюда вытекает [16], что $\Phi$ - ограниченное отображение банахова пространства $\mathfrak{T}\left(\mathscr{H}_{A}\right)$ в $\mathfrak{T}\left(\mathscr{H}_{B}\right)$. Отображение $\Phi^{*}$, двойственное к $\Phi$, однозначно определяется соотношением

$$
\operatorname{Tr} \Phi[X] Y=\operatorname{Tr} X \Phi^{*}[Y], \quad X \in \mathfrak{T}\left(\mathscr{H}_{A}\right), \quad Y \in \mathfrak{L}\left(\mathscr{H}_{B}\right),
$$

и называется сопряженным каналом. Сопряженный канал является линейным, вполне положительным *-слабо непрерывным отображением алгебры $\mathfrak{L}\left(\mathscr{H}_{B}\right)$ в $\mathfrak{L}\left(\mathscr{H}_{A}\right)$, унитальным в том смысле, что $\Phi\left[I_{\mathscr{H}_{B}}\right]=I_{\mathscr{H}_{A}}$. Здесь и далее $I$ с возможными индексами обозначает единичный оператор в соответствующем гильбертовом пространстве.

Существуют положительные, но не вполне положительные отображения, основной пример дается транспонированием матрицы $X \rightarrow X^{\top}$ в фиксированном базисе. 
Из определения полной положительности нетрудно получить [38], что композиция каналов $\Phi_{2} \circ \Phi_{1}$, определяемая как

$$
\Phi_{2} \circ \Phi_{1}[X]=\Phi_{2}\left[\Phi_{1}[X]\right]
$$

и естественно определенное тензорное произведение каналов

$$
\Phi_{1} \otimes \Phi_{2}=\left(\Phi_{1} \otimes \operatorname{Id}_{2}\right) \circ\left(\operatorname{Id}_{1} \otimes \Phi_{2}\right)
$$

являются каналами.

2.2. Представление типа Стайнспринга. Понятие вполне положительного отображения было введено Стайнспрингом [61] в более широком контексте $C^{*}$-алгебр. Оно охватывает также понятие гибридного канала, который имеет квантовый вход и классический выход или наоборот. Пример такого канала был упомянут в разделе 1. Мы не будем здесь развивать эту тему далее (см. [38]), а только отметим, что полная положительность сводится в таких случаях к положительности.

Отправляясь от знаменитой теоремы Наймарка о расширении, Стайнспринг установил представление вполне положительных отображений $C^{*}$-алгебр, которое в случае квантового канала [38] сводится к следующему.

ПреДЛОЖеНИЕ 2. Пусть $\Phi: A \rightarrow B-$ квантовый канал. Найдутся гильбертово пространство $\mathscr{H}_{E}$ и изометрический оператор $V: \mathscr{H}_{A} \rightarrow \mathscr{H}_{B} \otimes \mathscr{H}_{E}$ maкue, чmo

$$
\Phi[\rho]=\operatorname{Tr}_{E} V \rho V^{*}, \quad \rho \in \mathfrak{T}\left(\mathscr{H}_{A}\right),
$$

где $\operatorname{Tr}_{E}$ обозначает частичный след относительно $\mathscr{H}_{E}$. Представление (3) неединственно, однако любые два представления с операторами $V_{1}: \mathscr{H}_{A} \rightarrow$ $\mathscr{H}_{B} \otimes \mathscr{H}_{E_{1}}$ и $V_{2}: \mathscr{H}_{A} \rightarrow \mathscr{H}_{B} \otimes \mathscr{H}_{E_{2}}$ связаны посредством частичной изометрии $W: \mathscr{H}_{E_{1}} \rightarrow \mathscr{H}_{E_{2}}$ такой, что $V_{2}=\left(I_{B} \otimes W\right) V_{1} u V_{1}=\left(I_{B} \otimes W^{*}\right) V_{2}$.

Рассмотрим представление (3) для канала $\Phi$; комплементарный канал тогда определяется соотношением

$$
\widetilde{\Phi}[\rho]=\operatorname{Tr}_{B} V \rho V^{*}, \quad \rho \in \mathfrak{T}\left(\mathscr{H}_{A}\right) .
$$

Из соотношений между различными представлениями (3) вытекает, что комплементарный канал единствен в следующем смысле: любые два канала $\widetilde{\Phi}_{1}, \widetilde{\Phi}_{2}$, комплементарные к $\Phi$, изометрически эквивалентны в том смысле, что существует частичная изометрия $W: \mathscr{H}_{E_{1}} \rightarrow \mathscr{H}_{E_{2}}$ такая, что

$$
\widetilde{\Phi}_{2}[\rho]=W \widetilde{\Phi}_{1}[\rho] W^{*}, \quad \widetilde{\Phi}_{1}[\rho]=W^{*} \widetilde{\Phi}_{2}[\rho] W
$$

для всех $\rho$. Отсюда следует, что начальный проектор $W^{*} W$ удовлетворяет соотношению $\widetilde{\Phi}_{1}[\rho]=W^{*} W \widetilde{\Phi}_{1}[\rho]$, т. е. его носитель содержит носитель $\widetilde{\Phi}_{1}[\rho]$, тогда как конечный проектор $W W^{*}$ имеет аналогичное свойство по отношению к $\widetilde{\Phi}_{2}[\rho]$.

В общем случае мы называем два оператора плотности $\rho$ и $\sigma$ (возможно, определенные в разных гильбертовых пространствах) изометрически эквивалентными, если найдется частичная изометрия $W$ такая, что $\rho=W \sigma W^{*}$, 
$\sigma=W^{*} \rho W$. Очевидно, это имеет место тогда и только тогда, когда ненулевые собственные значения операторов плотности $\rho$ и $\sigma$ (с учетом кратности) совпадают. Мы обозначаем этот факт $\rho \sim \sigma$. Таким образом, мы только что показали, что $\widetilde{\Phi}_{1}[\rho] \sim \widetilde{\Phi}_{2}[\rho]$ для произвольного $\rho$. Можно показать, что канал, комплементарный к комплементарному каналу, изометрически эквивалентен исходному, так что $\Phi, \widetilde{\Phi}$ могут называться взаимно комплементарными.

Лемма 3. Пусть $\widetilde{\Phi}$ - комплементарный канал (4), тогда $\Phi\left[P_{\psi}\right] \sim \widetilde{\Phi}\left[P_{\psi}\right]$ для всех $\psi \in \mathscr{H}_{A}$.

ДоказАТЕЛЬство. Пусть $V: \mathscr{H}_{A} \rightarrow \mathscr{H}_{B} \otimes \mathscr{H}_{E}-$ изометрия из представления (3), (4), тогда $\rho_{B E}=V P_{\psi} V^{*}$ - чистое состояние в $\mathscr{H}_{B} \otimes \mathscr{H}_{E}$, и утверждение следует из фундаментального факта квантовой теории информации ("разложение Шмидта"): если $\rho_{B E}$ - чистое состояние в $\mathscr{H}_{B} \otimes \mathscr{H}_{E}$ и $\rho_{B}=\operatorname{Tr}_{E} \rho_{B E}$, $\rho_{E}=\operatorname{Tr}_{B} \rho_{B E}-$ его частичные состояния, то $\rho_{B} \sim \rho_{E}$ (см., например, [37; предложение 3]). Лемма доказана.

Другое название канала - динамическое отображение. В неравновесной статистической механике такие отображения возникают как результат необратимой эволюции открытой квантовой системы, взаимодействующей с окружением [38]. Предположим, что имеется составная квантовая система $A D=B E$ в гильбертовом пространстве

$$
\mathscr{H}=\mathscr{H}_{A} \otimes \mathscr{H}_{D} \simeq \mathscr{H}_{B} \otimes \mathscr{H}_{E}
$$

которая первоначально приготовлена в состоянии $\rho_{A} \otimes \rho_{D}$, а затем эволюционирует согласно унитарному оператору $U$ в $\mathscr{H}$. Тогда состояние выхода $\rho_{B}$ в зависимости от состояния входа $\rho_{A}=\rho$ имеет вид

$$
\Phi_{B}[\rho]=\operatorname{Tr}_{E} U\left(\rho \otimes \rho_{D}\right) U^{*},
$$

тогда как состояние “окружения” $E$ является выходным для канала

$$
\Phi_{E}[\rho]=\operatorname{Tr}_{B} U\left(\rho \otimes \rho_{D}\right) U^{*} .
$$

Если начальное состояние системы $D$ является чистым, $\rho_{D}=P_{\psi_{D}}$, то, вводя изометрию $V: \mathscr{H}_{A} \rightarrow \mathscr{H}_{B} \otimes \mathscr{H}_{D}$, которая действует как

$$
V \psi=U\left(\psi \otimes \psi_{D}\right), \quad \psi \in \mathscr{H}_{A},
$$

мы видим, что соотношения (7), (8) превращаются в $(3),(4)$, и канал $\Phi_{E}$ является комплементарным к $\Phi_{B}$. Отметим еще, что взятие частичного следа, а также унитарная эволюция очевидно являются вполне положительными операциями, откуда видно, что отображения (7), (8) вполне положительны; обратно, для всякого квантового канала существует представление такого вида (см., например, [38]).

Обширная литература посвящена изучению квантовых динамических полугрупп (некоммутативный аналог марковских полугрупп) и квантовых марковских процессов. Представление типа Стайнспринга (3) лежит в основе расширений квантовых динамических полугрупп до унитарной динамики открытой квантовой системы, взаимодействующей с окружением [16], [34]. 
2.3. Энтропийные количества и аддитивность. Рассмотрим норму отображения $\Phi$, определенную аналогично (1):

$$
\|\Phi\|_{1 \rightarrow p}=\sup _{X \neq 0} \frac{\|\Phi[X]\|_{p}}{\|X\|_{1}} \quad\left(=\sup _{\|X\|_{1} \leqslant 1}\|\Phi[X]\|_{p}\right),
$$

где $\|\cdot\|_{p}-p$-норма Шаттена [55]. Как показано в [4],

$$
\|\Phi\|_{1 \rightarrow p}^{p}=\sup _{\rho \in \mathfrak{S}_{\left(\mathscr{H}_{A}\right)}} \operatorname{Tr} \Phi[\rho]^{p}=\sup _{\psi \in \mathscr{H}_{A}} \operatorname{Tr} \Phi\left[P_{\psi}\right]^{p}
$$

где второе соотношение следует из выпуклости функции $x^{p}, p>1$.

Квантовая энтропия Реньи порядка $p>1$ оператора плотности $\rho$ определяется следующим образом:

$$
R_{p}(\rho)=\frac{1}{1-p} \log \operatorname{Tr} \rho^{p}=\frac{p}{1-p} \log \|\rho\|_{p} .
$$

Определим минимальную выходную энтропию Ренъи канала $\Phi$ :

$$
\check{R}_{p}(\Phi)=\inf _{\rho \in \mathfrak{S}(\mathscr{H})} R_{p}(\Phi[\rho])=\frac{p}{1-p} \log \|\Phi\|_{1 \rightarrow p},
$$

а также минимальную выходную энтропию фон Неймана:

$$
\check{H}(\Phi)=\inf _{\rho \in \mathfrak{S}(\mathscr{H})} H(\Phi[\rho]) .
$$

В пределе $p \rightarrow 1$ квантовые энтропии Реньи, монотонно не убывая, сходятся к энтропии фон Неймана:

$$
\lim _{p \rightarrow 1} R_{p}(\rho)=-\operatorname{Tr} \rho \log \rho=H(\rho) .
$$

В конечномерном случае множество квантовых состояний компактно, поэтому согласно лемме Дини минимальные выходные энтропии Реньи сходятся к минимальной выходной энтропии фон Неймана ${ }^{3}$.

Мультипликативность норм (9) для некоторых каналов $\Phi_{1}, \Phi_{2}$,

$$
\left\|\Phi_{1} \otimes \Phi_{2}\right\|_{1 \rightarrow p}=\left\|\Phi_{1}\right\|_{1 \rightarrow p} \cdot\left\|\Phi_{2}\right\|_{1 \rightarrow p},
$$

эквивалентна аддитивности минимальной выходной энтропии Реньи:

$$
\check{R}_{p}\left(\Phi_{1} \otimes \Phi_{2}\right)=\check{R}_{p}\left(\Phi_{1}\right)+\check{R}_{p}\left(\Phi_{2}\right) .
$$

С этим свойством тесно связана аддитивность минимальной выходной энтропии фон Неймана

$$
\check{H}\left(\Phi_{1} \otimes \Phi_{2}\right)=\check{H}\left(\Phi_{1}\right)+\check{H}\left(\Phi_{2}\right) .
$$

В конечномерном случае справедливость соотношения (15) для конкретных каналов $\Phi_{1}, \Phi_{2}$ и значений $p$, близких к 1 , влечет за собой (16) для этих каналов.

В последних двух соотношениях неравенство $\leqslant$ (аналогично неравенству $\geqslant$ в (14)) очевидно, поскольку правая часть равна инфимуму по подмножеству состояний-произведений $\rho=\rho_{1} \otimes \rho_{2}$. С другой стороны, именно наличие так называемых "сцепленных" чистых состояний, не сводящихся к состояниям-произведениям, и является причиной возможного нарушения равенства для квантовых каналов.

\footnotetext{
${ }^{3}$ Соответствующее утверждение неверно для бесконечномерных каналов (даже для классических каналов со счетным множеством состояний) - М. Е. Широков, устное сообщение.
} 
2.4. Пропускная способность канала. Практическая значимость свойства аддитивности (16) раскрывается в связи с понятием пропускной способности канала. Чтобы это объяснить проще, предположим на время, что пространства $\mathscr{H}_{A}, \mathscr{H}_{B}$ конечномерны.

Некоммутативный аналог шенноновской пропускной способности для квантового канала $\Phi$, который мы называем $\chi$-пропускной способностъю, определяется соотношением

$$
C_{\chi}(\Phi)=\sup _{\left\{\pi_{j}, \rho_{j}\right\}}\left(H\left(\Phi\left[\sum_{j} \pi_{j} \rho_{j}\right]\right)-\sum_{j} \pi_{j} H\left(\Phi\left[\rho_{j}\right]\right)\right),
$$

где супремум берется по всем квантовым ансамблям, т. е. конечным наборам состояний $\left\{\rho_{1}, \ldots, \rho_{n}\right\}$ с соответствующими вероятностями $\left\{\pi_{1}, \ldots, \pi_{n}\right\}$. Величина (17) тесно связана с пропускной способностью $C(\Phi)$ квантового канала $\Phi$ для передачи классической информации [37]. В общих чертах, классическая пропускная способность квантового канала определяется как максимальная скорость передачи при оптимальном выборе кодирований и декодирований для возрастающего числа $n$ независимых использований квантового канала

$$
\Phi^{\otimes n}=\underbrace{\Phi \otimes \cdots \otimes \Phi}_{n},
$$

при котором вероятность ошибки декодирования стремится к нулю при $n \rightarrow \infty$ (точное определение см. в [38]). Один из основных результатов квантовой теории информации, ХШВ-теорема ${ }^{4}[32]$, утверждает, что определенная таким образом пропускная способность $C(\Phi)$ выражается через $C_{\chi}(\Phi)$ по формуле

$$
C(\Phi)=\lim _{n \rightarrow \infty} \frac{1}{n} C_{\chi}\left(\Phi^{\otimes n}\right) .
$$

Поскольку величина $C_{\chi}(\Phi)$, как легко видеть, супераддитивна:

$$
C_{\chi}\left(\Phi_{1} \otimes \Phi_{2}\right) \geqslant C_{\chi}\left(\Phi_{1}\right)+C_{\chi}\left(\Phi_{2}\right),
$$

имеет место неравенство $C(\Phi) \geqslant C_{\chi}(\Phi)$. Однако если выполняется свойство аддитивности

$$
C_{\chi}\left(\Phi_{1} \otimes \Phi_{2}\right)=C_{\chi}\left(\Phi_{1}\right)+C_{\chi}\left(\Phi_{2}\right)
$$

для данного канала $\Phi_{1}=\Phi$ и произвольного канала $\Phi_{2}$, то

$$
C_{\chi}\left(\Phi^{\otimes n}\right)=n C_{\chi}(\Phi)
$$

откуда следует, что

$$
C(\Phi)=C_{\chi}(\Phi)
$$

Причиной возможного нарушения равенства здесь, как и в соотношениях (14), (15) и (16), является наличие сцепленных состояний, не сводимых к состояниям-произведениям, на входе тензорного произведения каналов $\Phi^{\otimes n}$.

\footnotetext{
${ }^{4}$ теорема Холево-Шумахера-Вестморленда.
} 
2.5. Основные выводы. Таким образом, естественно поставить вопрос: выполняется ли свойство аддитивности (16) глобально, т. е. для тензорного произведения любой пары квантовых каналов $\Phi_{1}, \Phi_{2}$ ? Формулировка этой проблемы восходит к работе [8] (см. также [37]). В замечательной работе Шоpa [60] (см. также [19]), была доказана эквивалентность свойств глобальной аддитивности $\chi$-пропускной способности и минимальной выходной энтропии.

Tеорема 4 [60]. Свойства (18) и (16) глобалъно эквивалентны в том смысле, что если одно из них выполняется для всех каналов $\Phi_{1}, \Phi_{2}$, то другое также выполняется для всех каналов.

Свойства аддитивности достаточно просто доказываются для всех классических каналов (см., например, [15]), однако в квантовом случае проблема оставалась открытой дюжину лет, и в конечном счете была решена в отрицательном смысле.

Подробная история вопроса вплоть до 2006 г. изложена в [37], и здесь мы только напомним основные этапы и обозначим конечные выводы. В работе [1] был предложен подход к изучению свойства аддитивности (16) через мультипликативность $(14)(1 \rightarrow p)$-норм (эквивалентную аддитивности (15) минимальных выходных энтропий Реньи). Первым явным примером, в котором это свойство нарушается для $d=\operatorname{dim} \mathscr{H} \geqslant 3$ и достаточно больших $p$, стал транспонированно-деполяризующий канал [64]:

$$
\Phi(\rho)=\frac{1}{d-1}\left[I \operatorname{Tr} \rho-\rho^{\top}\right]
$$

где $\rho \in \mathfrak{L}_{d}$ - матрица, а $\rho^{\top}-$ ее транспозиция. В частности, соотношение (15) с $\Phi_{1}=\Phi_{2}=\Phi$ не выполняется для $p \geqslant 4.7823$, если $d=3$ (тем не менее аддитивность величин $\check{H}(\Phi)$ и $C_{\chi}(\Phi)$ имеет место для этого канала). Через пять лет появились важные открытия Винтера [65] и Хайдена [28] (см. также [29]), которые доказали существование пар каналов с нарушением аддитивности минимальной выходной энтропии Реньи для всех значений параметра $p>1$. В основе этих и последующих работ лежит метод случайного розыгрыша каналов, которые для данных размерностей параметризуются изометриями $V$ в представлении (3), а также входных состояний каналов, и получение достаточно точных вероятностных оценок для норм типа (10). Для конечных размерностей соответствующие параметрические множества компактны и за основу обычно берется равномерное распределение. Опираясь на эти продвижения, Хастингс [26] дал доказательство существования каналов, для которых нарушается свойство аддитивности (16), формально соответствующее значению $p=1$, в очень высоких размерностях. При этом вероятность нарушения аддитивности стремится к 1 при неограниченном возрастании размерностей. Хастингс дал эскиз доказательства, а подробное изложение его подхода было затем дано Фукудой, Кингом и Мозером [18] и далее упрощено Брандау и Городецки [10]. Позднее в [3] было предложено доказательство, основанное на теореме Дворецкого-Мильмана о почти евклидовых сечениях многомерных выпуклых тел. 
В сочетании с теоремой 4 это дает вполне определенный отрицательный ответ на гипотезу аддитивности, однако ряд важных вопросов остается открытым. Все предложенные доказательства используют технику случайных унитарных каналов и случайных состояний и как таковые не являются конструктивными: они могут доказать только существование контрпримеров, но не позволяют их предъявить. Полученные на основе подхода Хастингса оценки размерностей, в которых проявляется неаддитивность, приводят к чрезвычайно высоким значениям: детальные оценки, проведенные в [18], дают $d \approx 10^{32}$ при нарушении аддитивности на величину порядка $10^{-5}$. Наилучшие результаты в этом направлении получены в [7], где утверждается, что "нарушение аддитивности минимальной выходной энтропии в модели случайных унитарных каналов и максимально сцепленного состояния возникает тогда и только тогда, когда выходное пространство имеет размерность не менее 183. При этом дефект аддитивности почти наверное меньше, чем $\log 2$, и может быть сделан сколь угодно близким к $\log 2 "$.

Хотя не исключена возможность лучших оценок, основывающихся на другой (но пока неизвестной) модели, это подвергает серьезному сомнению целесообразность поиска конкретных контрпримеров с помощью компьютерного моделирования случайных каналов. С этой точки зрения представляет интерес следующий явный пример, предложенный в [25]. Рассмотрим вполне положительное отображение

$$
\rho \rightarrow \Phi_{-}[\rho]=\operatorname{Tr}_{2} P_{-} \rho P_{-}, \quad \rho \in \mathfrak{T}(\mathscr{H} \otimes \mathscr{H}),
$$

где $P_{-}-$проектор на антисимметричное подпространство $\mathscr{H}_{-}$пространства $\mathscr{H} \otimes \mathscr{H}$, которое имеет размерность $d(d-1) / 2$, а частичный след берется относительно второй копии пространства $\mathscr{H}$. Ограничение этого отображения на операторы с носителем в подпространстве $\mathscr{H}_{-}$сохраняет след и, таким образом, является каналом. Можно показать [38], что $\Phi_{-}=((d-1) / 2) \widetilde{\Phi}^{*}$, где $\widetilde{\Phi}^{*}$ - сопряженный к комплементарному каналу для (21). Для этого простого канала минимальные энтропии Реньи неаддитивны при всех значениях $p>2$ и достаточно больших $d$, но, к сожалению, не известно, можно ли обобщить этот результат на наиболее интересный диапазон $p \geqslant 1$.

В общем случае остается неясным, что происходит в небольших размерностях: возможно, аддитивность все же имеет место по неизвестной причине, или же ее нарушение столь мало, что не может быть выявлено путем численного моделирования. Все это удивительно, учитывая тот факт, что физической причиной неаддитивности является сцепленность между входами параллельных квантовых каналов (см. более подробно в [38]).

С другой стороны, эти результаты подчеркивают важность продолжения усилий по нахождению специальных случаев, когда аддитивность по какой-то причине выполняется и может быть установлена аналитически.

Обзор основных классов таких "аддитивных" каналов, действующих в конечномерных пространствах, был представлен в [37]; ниже мы кратко перечислим наиболее важные классы каналов $\Phi$, для которых свойства аддитивности $(16),(18)$ и (15) при $p>1$ имеют место с $\Phi=\Phi_{1}$ и произвольным каналом $\Phi_{2}$. 
- q-битные унитальные каналь, т. е. каналы $\Phi: \mathfrak{L}_{2} \rightarrow \mathfrak{L}_{2}$, удовлетворяющие условию $\Phi[I]=I[45]$. Поразительно, что до сих пор нет аналитического доказательства аддитивности для неунитальных $q$-битных каналов, несмотря на убедительные численные подтверждения [27].

- Деполяризующий канал в $\mathfrak{L}_{d}$ :

$$
\Phi[\rho]=(1-p) \rho+p \frac{I}{d} \operatorname{Tr} \rho, \quad 0 \leqslant p \leqslant \frac{d^{2}}{d^{2}-1},
$$

который является единственным полностью унитарно-ковариантным каналом и который можно рассматривать как аналог вполне симметричного канала в классической теории информации [15]. Свойства аддитивности (16), (15), (18) были доказаны Кингом [46].

- Каналь, разрушающие сцепленность. В конечномерном случае это отображения вида

$$
\Phi[\rho]=\sum_{j} \rho_{B} \operatorname{Tr} \rho M_{A},
$$

где $\left\{M_{A}\right\}$ - разложение единицы в $\mathscr{H}_{A}: M_{A} \geqslant 0, \sum_{j} M_{A}=I_{A}$, и $\rho_{B} \in \mathfrak{S}\left(\mathscr{H}_{B}\right)$ (см. [43]).

Для конечномерных каналов, разрушающих сцепленность, аддитивность минимальной выходной энтропии и $\chi$-пропускной способности была установлена Шором [59], а аддитивность минимальной выходной энтропии Реньи - Кингом [44]. Свойства аддитивности каналов, разрушающих сцепленность, были обобщены на бесконечные размерности Широковым [57].

- Комплементарные каналы. Аддитивность минимальной выходной энтропии эквивалентна для канала $\Phi$ и его комплементарного $\widetilde{\Phi}$ (см. ниже лемму 5 ). Класс каналов, комплементарных к каналам, разрушающим сцепленность, содержит операцию умножения Шура для матриц $\rho=\left[c_{j k}\right]_{j, k=1, \ldots, d}$ в $\mathfrak{L}_{d}$ :

$$
\widetilde{\Phi}[\rho]=\left[\gamma_{j k} c_{j k}\right]_{j, k=1, \ldots, d},
$$

где $\left[\gamma_{j k}\right]_{j, k=1, \ldots, d}$ - фиксированная неотрицательно определенная матрица такая, что $\gamma_{j j} \equiv 1$. Для таких каналов, называемых также каналами Адамара, была установлена и аддитивность $\chi$-пропускной способности [47].

В следующих разделах мы рассмотрим бозонные гауссовские каналы, которые действуют в бесконечномерных пространствах. Имеется гипотеза, что все свойства аддитивности выполняются в этом классе каналов. Одной из основных целей настоящей работы является доказательство того факта, что аддитивность выполняется для широкого класса калибровочно-ковариантных или контравариантных гауссовских каналов, т. е. тех, которые согласованы с фиксированной комплексной структурой в соответствующем симплектическом пространстве.

2.6. Мажоризация для квантовых состояний. С этого момента мы вновь допускаем к рассмотрению бесконечномерные гильбертовы пространства. Обозначим $\mathfrak{F}$ класс вещественных вогнутых функций $f$ на отрезке $[0,1]$ 
таких, что $f(0)=0$. Для любой $f \in \mathfrak{F}$ и любого оператора плотности $\rho$ можно определить величину

$$
\operatorname{Tr} f(\rho)=\sum_{j} f\left(\lambda_{j}\right)
$$

где $\lambda_{j}$ - ненулевые собственные значения оператора плотности $\rho$ с учетом кратности. Отметим, что эта величина определена однозначно и принимает значения в $(-\infty, \infty]$. Это следует из того, что $f(x) \geqslant c x$, где $c=f(1)$, значит, $\operatorname{Tr} f(\rho) \geqslant c \operatorname{Tr} \rho=c$. Мы также будем использовать тот факт, что функционал $\rho \rightarrow \operatorname{Tr} f(\rho)$ является (строго) вогнутым на $\mathfrak{S}(\mathscr{H})$, если $f-($ строго) вогнута (см., например, [11]).

Обозначим $\lambda_{j}^{\downarrow}(\rho)$ собственные значения оператора плотности $\rho$ с учетом кратности, упорядоченные в порядке невозрастания. Оператор плотности $\rho$ мажоpupyem оператор плотности $\sigma$, если

$$
\sum_{j=1}^{k} \lambda_{j}^{\downarrow}(\rho) \geqslant \sum_{j=1}^{k} \lambda_{j}^{\downarrow}(\sigma), \quad k=1,2, \ldots
$$

Из хорошо известного результата (см., например, [11]) вытекает, что такая мажоризация имеет место тогда и только тогда, когда $\operatorname{Tr} f(\rho) \leqslant \operatorname{Tr} f(\sigma)$ для всех $f \in \mathfrak{F}$.

Для данного квантового канала $\Phi$ введем величину

$$
\check{f}(\Phi)=\inf _{\rho \in \mathfrak{S}(\mathscr{H})} \operatorname{Tr} f(\Phi[\rho])=\inf _{P_{\psi} \in \mathfrak{P}(\mathscr{H})} \operatorname{Tr} f\left(\Phi\left[P_{\psi}\right]\right)
$$

где второе равенство следует из вогнутости функционала $\rho \rightarrow \operatorname{Tr} f(\Phi[\rho])$ на $\mathfrak{S}(\mathscr{H})$. Более того, для строго выпуклой $f$ всякий минимизатор имеет форму $P_{\psi}$ для некоторого единичного вектора $\psi \in \mathscr{H}$.

В частности, полагая $f(x)=-x \log x$ или $f(x)=-x^{p}$, мы получаем $\check{f}(\Phi)=$ $\check{H}(\Phi)$ или $\check{f}(\Phi)=-\|\Phi\|_{1 \rightarrow p}^{p}$.

Лемма 5 [36]. Для комплементарных каналов выполнено равенство $\check{f}(\Phi)=$ $\check{f}(\widetilde{\Phi})$. Таким образом, $\|\Phi\|_{1 \rightarrow p}=\|\widetilde{\Phi}\|_{1 \rightarrow p}, \check{H}(\Phi)=\check{H}(\widetilde{\Phi}), \check{R}_{p}(\Phi)=\check{R}_{p}(\widetilde{\Phi})$ и мультипликативность (14), а также аддитивность минимальных выходных энтропий (16), (15) выполняется одновременно для пар каналов $\Phi_{1}, \Phi_{2}$ и $\widetilde{\Phi}_{1}, \widetilde{\Phi}_{2}$.

ДокАЗАТЕльСтво. Согласно лемме 3 , операторы $\Phi\left[P_{\psi}\right]$ и $\widetilde{\Phi}\left[P_{\psi}\right]$ имеют одинаковый ненулевой спектр $\left(\Phi\left[P_{\psi}\right] \sim \widetilde{\Phi}\left[P_{\psi}\right]\right)$. Следовательно,

$$
\operatorname{Tr} f\left(\Phi\left[P_{\psi}\right]\right)=\operatorname{Tr} f\left(\widetilde{\Phi}\left[P_{\psi}\right]\right)
$$

поскольку $f(0)=0$. Используя второе равенство в $(22)$, получаем $\check{f}(\Phi)=\check{f}(\widetilde{\Phi})$.

Утверждение о мультипликативности (аддитивности) является следствием того факта, что канал $\widetilde{\Phi}_{1} \otimes \widetilde{\Phi}_{2}$ комплементарен к $\Phi_{1} \otimes \Phi_{2}$. Лемма доказана. 


\section{3. Квантовые гауссовские системы}

3.1. Гауссовские состояния и каналы. Вещественное векторное пространство $Z$, снабженное невырожденной кососимметричной формой $\Delta\left(z, z^{\prime}\right)$, называется симплектическим пространством. Всюду далее $Z$ конечномерно, тогда его размерность с необходимостью является четной, $\operatorname{dim} Z=2 s$ [49]. Базис $\left\{e_{j}, h_{j} ; j=1, \ldots, s\right\}$, в котором форма $\Delta\left(z, z^{\prime}\right)$ имеет матрицу

$$
\Delta=\operatorname{diag}\left[\begin{array}{cc}
0 & 1 \\
-1 & 0
\end{array}\right]_{j=1, \ldots, s},
$$

называется симплектическим. Системой Вейля в гильбертовом пространстве $\mathscr{H}$ называется сильно непрерывное семейство унитарных операторов $\{W(z) ; z \in Z\}$, удовлетворяющее каноническим коммутационным соотношениям (КKC) Вейля-Сигала

$$
W(z) W\left(z^{\prime}\right)=\exp \left\{-\frac{i}{2} \Delta\left(z, z^{\prime}\right)\right\} W\left(z+z^{\prime}\right) .
$$

Таким образом, $z \rightarrow W(z)$ является проективным представлением аддитивной группы $Z$. Мы будем рассматривать только неприводимые представления. Теорема единственности Стоуна-фон Неймана утверждает, что такое представление единственно с точностью до унитарной эквивалентности. Хорошо известно (см., например, [55]), что имеется семейство самосопряженных операторов $z \rightarrow R(z)$ с общей существенной областью определения $\mathscr{D}$ такое, что

$$
W(z)=\exp \{i R(z)\}
$$

более того, в симплектическом базисе $\left\{e_{j}, h_{j} ; j=1, \ldots, s\right\}$

$$
R(z)=\sum_{j=1}^{s}\left(x_{j} q_{j}+y_{j} p_{j}\right)
$$

на $\mathscr{D}$, где $R\left(e_{j}\right)=q_{j}, R\left(h_{j}\right)=p_{j}$, а $\left[x_{1}, y_{1}, \ldots, x_{s}, y_{s}\right]$ - координаты вектора $z$ в этом базисе. Здесь канонические наблюдаемье $q_{j}, p_{j}, j=1, \ldots, s,-$ самосопряженные операторы в $\mathscr{H}$, удовлетворяющие $\mathrm{KKC}$ Гейзенберга на $\mathscr{D}$ :

$$
\left[q_{j}, p_{k}\right] \subseteq i \delta_{j k} I, \quad\left[q_{j}, q_{k}\right]=0, \quad\left[p_{j}, p_{k}\right]=0 .
$$

В физике симплектическое пространство является фазовым пространством классической системы (такой как набор мод электромагнитного поля в полости), квантовая версия которой описывается KKC. Тогда $s$ является числом степеней свободы или "нормальных мод" классической системы.

Состояние, задаваемое оператором плотности $\rho$ в $\mathscr{H}$, называется гауссовским, если его квантовая характеристическая функиия

$$
\phi(z)=\operatorname{Tr} \rho W(z)
$$

имеет вид

$$
\phi(z)=\exp \left\{i m(z)-\frac{1}{2} \alpha(z, z)\right\}
$$


где $m$ - вещественная линейная форма, а $\alpha$ - вещественная билинейная симметричная форма на $Z$. Необходимое и достаточное условие для того, чтобы соотношение (27) определяло состояние, состоит в неотрицательной определенности (комплексной) эрмитовой формы ${ }^{5} \alpha\left(z, z^{\prime}\right)-(i / 2) \Delta\left(z, z^{\prime}\right)$ на $Z$ или, кратко:

$$
\alpha \geqslant \frac{i}{2} \Delta
$$

Условимся, что матрица билинейной формы в фиксированном симплектическом базисе будет обозначаться той же буквой, что и сама форма, тогда соотношение (28) можно понимать как неравенство для эрмитовых матриц, где $\alpha-$ вещественная симметричная, а $\Delta$ - вещественная кососимметричная матрица.

Гауссовское состояние является чистым тогда и только тогда, когда $\alpha$ является минимальным решением этого неравенства (см., например, [39]). Оператор $J$ в $Z$ называется оператором комплексной структуры, если

$$
J^{2}=-I,
$$

где $I$ - единичный оператор в $Z$, и билинейная форма $\Delta\left(z, J z^{\prime}\right)$ является (евклидовым) скалярным произведением в $Z$, т. е.

$$
\begin{gathered}
\Delta\left(z, J z^{\prime}\right)=\Delta\left(z^{\prime}, J z\right) \quad\left(=-\Delta\left(J z, z^{\prime}\right)\right) ; \\
\Delta(z, J z) \geqslant 0, \quad z \in Z .
\end{gathered}
$$

Следующая характеризация содержится в работах [17], [38].

ПрЕДЛожЕниЕ 6. Минимальные решения неравенства (28) находятся во взаимно однозначном соответствии с операторами $J$ комплексной структурьє в $Z$, которое задается соотношением

$$
\alpha\left(z, z^{\prime}\right)=\frac{1}{2} \Delta\left(z, J z^{\prime}\right), \quad z, z^{\prime} \in Z .
$$

Таким образом, каждой комплексной структуре соответствует семейство чистых гауссовских состояний (27), параметризованное различными значениями $m$, которые называются $J$-когерентными состояниями. Состояние с $m=0$ называется $J$-вакуумом. Если $\rho_{0}$ - вакуум, то соответствующие когерентные состояния имеют вид $W\left(z^{\prime}\right) \rho_{0} W\left(z^{\prime}\right)^{*}$, что следует из соотношения

$$
W\left(z^{\prime}\right)^{*} W(z) W\left(z^{\prime}\right)=\exp \left\{-i \Delta\left(z, z^{\prime}\right)\right\} W(z)
$$

и невырожденности формы $\Delta\left(z, z^{\prime}\right)$, благодаря которой всякая линейная форма на $Z$ имеет вид $m(z)=\Delta\left(z, z_{m}^{\prime}\right)$.

Оператор $S$ в $Z$ называется симплектическим, если $\Delta\left(S z, S z^{\prime}\right)=\Delta\left(z, z^{\prime}\right)$ для всех $z, z^{\prime} \in Z$. Унитарные операторы $W(S z)$ удовлетворяют $\operatorname{KKC}(25)$, поэтому в силу теоремы единственности Стоуна-фон Неймана найдется унитарный оператор $U_{S}$ в $\mathscr{H}$ такой, что

$$
W(S z)=U_{S}^{*} W(z) U_{S}, \quad z \in Z .
$$

\footnotetext{
${ }^{5}$ Комплекснозначная вещественно-билинейная форма $\beta\left(z, z^{\prime}\right)$ на $Z$ будет называться эрмитовой, если $\beta\left(z^{\prime}, z\right)=\overline{\beta\left(z, z^{\prime}\right)}$.
} 
Отображение $S \rightarrow U_{S}$ является проективным представлением группы всех симплектических преобразований $Z$, иногда называемым “метаплектическим представлением" [2], поскольку оно может быть продолжено до точного унитарного представления метаплектической группы, которая является двукратной накрывающей симплектической группы.

Аналогичным образом, оператор $T$ называется антисимплектическим, если $\Delta\left(T z, T z^{\prime}\right)=-\Delta\left(z, z^{\prime}\right)$ для всех $z, z^{\prime} \in Z$. Найдется антиунитарный оператор $U_{T}$ в $\mathscr{H}$ такой, что

$$
W(T z)=U_{T}^{*} W(z) U_{T}, \quad z \in Z .
$$

Пусть $Z_{A}, Z_{B}$ - два симплектических пространства с соответствующими системами Вейля. Канал $\Phi: A \rightarrow B$ называется гауссовским, если сопряженный канал удовлетворяет соотношению

$$
\Phi^{*}\left[W_{B}(z)\right]=W_{A}(K z) \exp \left\{i l(z)-\frac{1}{2} \mu(z, z)\right\}, \quad z \in Z_{B},
$$

где $K: Z_{B} \rightarrow Z_{A}$ - линейный оператор, $l$ - линейная форма и $\mu$ - вещественная симметричная форма на $Z_{B}$. В терминах характеристических функций состояний,

$$
\phi_{B}(z)=\phi_{A}(K z) \exp \left\{i l(z)-\frac{1}{2} \mu(z, z)\right\} .
$$

Отсюда видно, что гауссовский канал отображает гауссовские состояния в гауссовские состояния. Обратное утверждение также верно [17]. Канал называется центрированнъм, если $l=0$.

Необходимое и достаточное условие на параметры $(K, l, \mu)$ для полной положительности отображения $\Phi$ состоит в неотрицательной определенности эрмитовой формы

$$
z, z^{\prime} \rightarrow \mu\left(z, z^{\prime}\right)-\frac{i}{2}\left[\Delta_{B}\left(z, z^{\prime}\right)-\Delta_{A}\left(K z, K z^{\prime}\right)\right]
$$

на $Z_{B}$ или, в терминах матриц (если в $Z_{A}, Z_{B}$ выбраны некоторые базисы), в выполнении неравенства

$$
\mu \geqslant \frac{i}{2}\left(\Delta_{B}-K^{t} \Delta_{A} K\right)
$$

где ${ }^{t}$ обозначает транспонирование матрицы. Доказательство этого факта, использующее явное построение представления вида (7), дано в [14] (см. также [38]); ниже, в предложении 12, мы дадим такое построение для важного частного класса гауссовских каналов.

Мы называем гауссовский канал экстремальным ${ }^{6}$, если $\mu$ является минимальным решением неравенства (33). Эта терминология мотивируется тем, что минимальность $\mu$ оказывается необходимой и достаточной для того, чтобы канал $\Phi$ являлся крайней точкой выпуклого множества всех каналов с фиксированными входом и выходом [39].

\footnotetext{
${ }^{6} \mathrm{~B}$ квантовой оптике говорят о квантово-ограниченных каналах [20].
} 
Гипотеза аддитивности для квантовых гауссовских каналов: свойства аддитивности (15), (16) выполняются для любой пары гауссовских каналов $\Phi_{1}, \Phi_{2}$.

Гипотеза о квантовых гауссовских минимизаторах: для любой функции $f \in \mathfrak{F}$ инфимум в соотношении (22) достигается на чистом гауссовском состоянии $\rho$.

Всякий гауссовский канал имеет свойство ковариантности

$$
\Phi\left[W_{A}(z) \rho W_{A}(z)^{*}\right]=W_{B}\left(K^{s} z\right) \Phi[\rho] W_{B}\left(K^{s} z\right)^{*},
$$

где $K^{s}$ - симплектически сопряженный оператор, определяемый соотношением

$$
\Delta_{B}\left(K^{s} z_{A}, z_{B}\right)=\Delta_{A}\left(z_{A}, K z_{B}\right) .
$$

Отсюда следует, что величина $\operatorname{Tr} f(\Phi[\rho])$ имеет одно и то же значение для всех когерентных состояний $W(z) \rho_{0} W(z)^{*}$, ассоциированных с данным вакуумным состоянием $\rho_{0}$.

Эти две гипотезы оказываются тесно связанными. Далее мы опишем их положительное решение для специального и важного класса гауссовских каналов с калибровочной симметрией. Однако обе гипотезы остаются открытыми для произвольных гауссовских каналов.

3.2. Комплексные структуры и калибровочная симметрия. Если в пространстве $Z$ задан оператор комплексной структуры $J$, то можно определить евклидово скалярное произведение $j\left(z, z^{\prime}\right)=\Delta\left(z, J z^{\prime}\right)$. Таким образом, в $Z$ определена структура $s$-мерного унитарного пространства $\mathbf{Z}$, в котором $i \mathbf{z}$ соответствует $J z$, а соответствующее (эрмитово) скалярное произведение дается формулой ${ }^{7}$

$$
\mathbf{j}\left(\mathbf{z}, \mathbf{z}^{\prime}\right)=\frac{1}{2}\left[\Delta\left(z, J z^{\prime}\right)+i \Delta\left(z, z^{\prime}\right)\right]=\frac{1}{2}\left[j\left(z, z^{\prime}\right)-i j\left(z, J z^{\prime}\right)\right] .
$$

Из соотношений $(29),(30)$ следует, что оператор $J$ - симплектический, т. е. $\Delta\left(J z, J z^{\prime}\right)=\Delta\left(z, z^{\prime}\right)$ для всех $z, z^{\prime} \in Z$. С каждой комплексной структурой можно ассоциировать циклическую однопараметрическую группу симплектических преобразований $\left\{\mathrm{e}^{\varphi J} ; \varphi \in[0,2 \pi)\right\}$, называемую калибровочной группой. Согласно теореме единственности Стоуна-фон Неймана, калибровочная группа в $Z$ порождает однопараметрическую унитарную группу калибровочнъх преобразований $\left\{U_{\varphi} ; \varphi \in[0,2 \pi)\right\}$ в $\mathscr{H}$ согласно формуле

$$
W\left(\mathrm{e}^{\varphi J} z\right)=U_{\varphi}^{*} W(z) U_{\varphi}
$$

Для будущих целей удобно ввести комплексную параметризацию системы Вейля, введя операторы смещения

$$
D(\mathbf{z})=W(J z), \quad \mathbf{z} \in \mathbf{Z} .
$$

\footnotetext{
${ }^{7}$ В соответствии с соглашением, принятым в математической физике, скалярное произведение комплексно-линейно по аргументу $\mathbf{z}^{\prime}$ и антилинейно по $\mathbf{z}$.
} 
Состояние $\rho$ является калибровочно-инвариантным, если $\rho=U_{\varphi} \rho U_{\varphi}^{*}$ для всех $\varphi$, что эквивалентно свойству $\phi(z)=\phi\left(\mathrm{e}^{\varphi J} z\right)$ характеристической функции. В частности, гауссовское состояние (27) калибровочно-инвариантно, если $m(z) \equiv 0$ и $\alpha\left(z, z^{\prime}\right)=\alpha\left(J z, J z^{\prime}\right)$. Вводя в $\mathbf{Z}$ эрмитово скалярное произведение

$$
\boldsymbol{\alpha}\left(\mathbf{z}, \mathbf{z}^{\prime}\right)=\frac{1}{2}\left[\alpha\left(z, z^{\prime}\right)-i \alpha\left(z, J z^{\prime}\right)\right]
$$

имеем $\boldsymbol{\alpha}(\mathbf{z}, \mathbf{z})=\alpha(z, z) / 2$, поскольку $\alpha\left(z, J z^{\prime}\right)$ кососимметрична; далее, условие (28) эквивалентно неотрицательной определенности эрмитовой формы $\boldsymbol{\alpha}\left(\mathbf{z}, \mathbf{z}^{\prime}\right)-\mathbf{j}\left(\mathbf{z}, \mathbf{z}^{\prime}\right) / 2$ на $\mathbf{Z}:$

$$
\alpha \geqslant \frac{1}{2} \mathbf{j}
$$

Это получается применением приводимой ниже леммы 7 к форме

$$
z, z^{\prime} \rightarrow \beta\left(z, z^{\prime}\right)=\alpha\left(z, z^{\prime}\right)-\frac{i}{2} \Delta\left(z, z^{\prime}\right)
$$

Соотношение (37) может рассматриваться как неравенство для матриц соответствующих форм, если в $\mathbf{Z}$ выбран базис. Для ортонормированного базиса $\mathbf{j}=\mathbf{I}-$ единичная матрица.

Лемма 7. Пусть $\beta\left(z, z^{\prime}\right)$ - билинейная, комплекснозначная эрмитова форма на вещественном векторном пространстве $Z$, удовлетворяющая условию $\beta\left(J z, J z^{\prime}\right)=\beta\left(z, z^{\prime}\right)$, где $J$ - линейный оператор такой, что $J^{2}=-I$. Тогда $\beta\left(z, z^{\prime}\right)$ неотрицательно определена, т.е.

$$
\sum_{j, k} \bar{c}_{j} c_{k} \beta\left(z_{j}, z_{k}\right) \geqslant 0
$$

для любого конечного набора $\left\{z_{j}\right\} \subset Z$ и любых чисел $\left\{c_{j}\right\} \subset \mathbb{C}$, тогда и только тогда, когда

$$
\operatorname{Re} \beta(z, z) \pm \operatorname{Im} \beta(z, J z) \geqslant 0 \quad \text { для всех } z \in Z .
$$

Доказательство. $(39) \Rightarrow(38)$. Имеем $\beta\left(z, z^{\prime}\right)=\operatorname{Re} \beta\left(z, z^{\prime}\right)+i \operatorname{Im} \beta\left(z, z^{\prime}\right)$, где $\operatorname{Im} \beta\left(z, z^{\prime}\right)$ кососимметрична, поэтому $\operatorname{Im} \beta(z, z)=0$. Используя тот факт, что $\beta\left(J z, z^{\prime}\right)=-\beta\left(z, J z^{\prime}\right)$, получаем также, что форма $\operatorname{Re} \beta\left(z, J z^{\prime}\right)$ кососимметрична, поэтому $\operatorname{Re} \beta(z, J z)=0$. Таким образом,

$$
\operatorname{Re} \beta(z, z) \pm \operatorname{Im} \beta(z, J z)=\beta(z, z) \mp i \beta(z, J z) .
$$

Теперь введем комплексификацию $z \leftrightarrow \mathbf{z}$, полагая $J z \leftrightarrow i \mathbf{z}$, и определим две эрмитовы формы на комплексификации $\mathbf{Z}$ пространства $Z$ :

$$
\boldsymbol{\beta}^{\mp}\left(\mathbf{z}, \mathbf{z}^{\prime}\right)=\beta\left(z, z^{\prime}\right) \mp i \beta\left(z, J z^{\prime}\right) .
$$

Тогда форма $\boldsymbol{\beta}^{-}$полуторалинейна (т. е. комплексно-линейна по аргументу $\mathbf{z}^{\prime}$ и антилинейна по z), тогда как $\boldsymbol{\beta}^{+}$антиполуторалинейна. Из (40), (39) следует, что

$$
\boldsymbol{\beta}^{\mp}(\mathbf{z}, \mathbf{z})=\operatorname{Re} \beta(z, z) \pm \operatorname{Im} \beta(z, J z) \geqslant 0 \quad \text { для всех } \mathbf{z} \in \mathbf{Z},
$$


поэтому в силу (анти-)полуторалинейности

$$
\sum_{j, k} \bar{c}_{j} c_{k} \boldsymbol{\beta}^{\mp}\left(\mathbf{z}_{j}, \mathbf{z}_{k}\right) \geqslant 0 .
$$

Складывая два неравенства, отвечающие плюсу и минусу, получаем (38).

$(38) \Rightarrow(39)$. Применяя $(38)$ к набору $\left\{z_{j}, J z_{j}\right\} \subset Z,\left\{c_{j}, \pm i c_{j}\right\} \subset \mathbb{C}$, получаем

$$
\sum_{j, k} \bar{c}_{j} c_{k}\left[\beta\left(z_{j}, z_{k}\right) \pm i \beta\left(z_{j}, J z_{k}\right)\right] \geqslant 0
$$

поэтому формы (40) неотрицательно определены. В силу (анти-)полуторалинейности этих форм, это эквивалентно соотношению (41), т. е. (39). Лемма доказана.

Предположим, что в $Z_{A}, Z_{B}$ зафиксированы операторы комплексной структуры $J_{A}, J_{B}$, и пусть $U_{\phi}^{A}, U_{\phi}^{B}$ - соответствующие калибровочные преобразования в $\mathscr{H}_{A}, \mathscr{H}_{B}$, действующие согласно (35). Канал $\Phi: A \rightarrow B$ называется калибровочно-ковариантным, если

$$
\Phi\left[U_{\phi}^{A} \rho\left(U_{\phi}^{A}\right)^{*}\right]=U_{\phi}^{B} \Phi[\rho]\left(U_{\phi}^{B}\right)^{*}
$$

для всех входных состояний $\rho$ и всех $\phi \in[0,2 \pi]$. Для гауссовского канала (32) с параметрами $(K, l, \mu)$ это сводится к

$$
l(z) \equiv 0, \quad K J_{B}-J_{A} K=0, \quad \mu\left(z, z^{\prime}\right)=\mu\left(J_{B} z, J_{B} z^{\prime}\right) .
$$

Соотношение (32) для гауссовских калибровочно-ковариантных каналов принимает следующий вид (ср. [30]):

$$
\Phi^{*}\left[D_{B}(\mathbf{z})\right]=D_{A}(\mathbf{K z}) \exp \{-\boldsymbol{\mu}(\mathbf{z}, \mathbf{z})\}, \quad \mathbf{z} \in \mathbf{Z}_{B},
$$

где

$$
\boldsymbol{\mu} \geqslant \pm \frac{1}{2}\left[\mathbf{j}_{B}-\mathbf{K}^{*} \mathbf{j}_{A} \mathbf{K}\right]
$$

Эквивалентность соотношений (44) и (33) получается путем применения леммы 7 к эрмитовой форме

$$
\beta\left(z, z^{\prime}\right)=\mu\left(z, z^{\prime}\right)-\frac{i}{2}\left[\Delta_{B}\left(z, z^{\prime}\right)-\Delta_{A}\left(K z, K z^{\prime}\right)\right] .
$$

Канал $\Phi: A \rightarrow B$ называется калибровочно-контравариантным, если

$$
\Phi\left[U_{\phi}^{A} \rho\left(U_{\phi}^{A}\right)^{*}\right]=\left(U_{\phi}^{B}\right)^{*} \Phi[\rho] U_{\phi}^{B}
$$

для всех входных состояний $\rho$ и всех $\phi \in[0,2 \pi]$. Для гауссовского канала (32) с параметрами $(K, l, \mu)$ это сводится к

$$
l(z) \equiv 0, \quad K J_{B}+J_{A} K=0, \quad \mu\left(z, z^{\prime}\right)=\mu\left(J_{B} z, J_{B} z^{\prime}\right) .
$$

Соотношение (32) для калибровочно-контравариантного канала принимает вид

$$
\Phi^{*}\left[D_{B}(\mathbf{z})\right]=D_{A}(-\Lambda \mathbf{K} \mathbf{z}) \exp \{-\boldsymbol{\mu}(\mathbf{z}, \mathbf{z})\}, \quad \mathbf{z} \in \mathbf{Z}_{B},
$$


где $\Lambda$ - антилинейный оператор комплексного сопряжения, $\Lambda^{2}=I, \Lambda^{s}=-\Lambda$ в $\mathbf{Z}_{A}$, так что $\Lambda J_{A}+J_{A} \Lambda=0$, и $\mathbf{K}=-\Lambda K$ - комплексно-линейный оператор из $\mathbf{Z}_{B}$ в $\mathbf{Z}_{A}$. Здесь

$$
\boldsymbol{\mu} \geqslant \pm \frac{1}{2}\left[\mathbf{j}_{B}+\mathbf{K}^{*} \mathbf{j}_{A} \mathbf{K}\right]
$$

Последнее условие получается применением леммы 7 к эрмитовой форме

$$
\begin{aligned}
\beta\left(z, z^{\prime}\right) & =\mu\left(z, z^{\prime}\right)-\frac{i}{2}\left[\Delta_{B}\left(z, z^{\prime}\right)-\Delta_{A}\left(K z, K z^{\prime}\right)\right] \\
& =\frac{i}{2}\left[\Delta_{B}\left(z, z^{\prime}\right)+\Delta_{A}\left(K z, K z^{\prime}\right)\right] .
\end{aligned}
$$

3.3. Аттенюаторы и усилители. В дальнейшем мы ограничиваем наши рассмотрения гауссовскими каналами, которые калибровочно-ковариантны либо контравариантны. Поэтому, желая быть более конкретными, мы будем рассматривать векторы в $\mathbf{Z}$ как $s$-мерные комплексные векторы-столбцы, причем оператор $J$ действует как умножение на $i$, соответствующее эрмитово скалярное произведение есть $\mathbf{j}\left(\mathbf{z}, \mathbf{z}^{\prime}\right)=\mathbf{z}^{*} \mathbf{z}^{\prime}$, а симплектическая форма имеет вид $\Delta\left(z, z^{\prime}\right)=2 \operatorname{Im} \mathbf{z}^{*} \mathbf{z}^{\prime}$, где * обозначает эрмитово сопряжение. Линейные операторы в $\mathbf{Z}$, коммутирующие с $J$, представляются комплексными $(s \times s)$-матрицами. Калибровочная группа действует в $\mathbf{Z}$ как умножение на $\mathrm{e}^{i \phi}$. Гауссовские калибровочно-инвариантные состояния описываются модифицированными характеристическими функциями

$$
\phi(\mathbf{z})=\operatorname{Tr} \rho D(\mathbf{z})=\exp \left\{-\mathbf{z}^{*} \boldsymbol{\alpha} \mathbf{z}\right\}
$$

где $\boldsymbol{\alpha}$ - эрмитова корреляционная матрица, удовлетворяющая неравенству $\boldsymbol{\alpha} \geqslant$ $\mathbf{I} / 2$, которое вытекает из (37). Для данной комплексной структуры единственным минимальным решением этого неравенства является $\mathbf{I} / 2$, чему соответствуют вакуумное состояние $\rho_{0}$ и семейство когерентных состояний $\left\{\rho_{\mathbf{z}} ; \mathbf{z} \in \mathbf{Z}\right\}$, так что $\rho_{\mathbf{z}}=D(\mathbf{z}) \rho_{0} D(\mathbf{z})^{*}$. Имеем

$$
\operatorname{Tr} \rho_{\mathbf{w}} D(\mathbf{z})=\exp \left\{2 i \operatorname{Im} \mathbf{w}^{*} \mathbf{z}-\frac{1}{2}|\mathbf{z}|^{2}\right\},
$$

где $|\mathbf{z}|^{2}=\mathbf{z}^{*} \mathbf{z}$.

Пусть $\mathbf{Z}_{A}, \mathbf{Z}_{B}$ - входное и выходное пространства размерностей $s_{A}, s_{B}$ соответственно. Мы обозначаем $s_{A}=\operatorname{dim} \mathbf{Z}_{A}, s_{B}=\operatorname{dim} \mathbf{Z}_{B}$ число мод на входе и на выходе канала. Действие гауссовского калибровочно-ковариантного канала (43) может быть описано соотношением

$$
\Phi^{*}\left[D_{B}(\mathbf{z})\right]=D_{A}(\mathbf{K z}) \exp \left\{-\mathbf{z}^{*} \boldsymbol{\mu} \mathbf{z}\right\}, \quad \mathbf{z} \in \mathbf{Z}_{B},
$$

где $\mathbf{K}-$ комплексная $\left(s_{B} \times s_{A}\right)$-матрица, $\boldsymbol{\mu}$ - эрмитова $\left(s_{B} \times s_{B}\right)$-матрица и выполнены неравенства

$$
\boldsymbol{\mu} \geqslant \pm \frac{1}{2}\left(\mathbf{I}_{B}-\mathbf{K}^{*} \mathbf{K}\right)
$$

где $\mathbf{I}_{B}-$ единичная $\left(s_{B} \times s_{B}\right)$-матрица. Это следует из (44), принимая во внимание, что матрица формы $\mathbf{j}\left(\mathbf{z}, \mathbf{z}^{\prime}\right)$ в ортонормированном базисе совпадает с единичной матрицей $\mathbf{I}$ соответствующей размерности. В дальнейшем нам понадобится следующая лемма. 
Лемма 8. Отображение (49) является инбективным тогда и только тогда, когда $\mathbf{K}^{8} \mathbf{K}^{*}>0$ (в этом случае с необходимостъю $s_{B} \geqslant s_{A}$ ).

ДокАЗАТЕЛЬСтво. Инъективность означает, что из равенства $\Phi\left[\rho_{1}\right]=\Phi\left[\rho_{2}\right]$ следует равенство $\rho_{1}=\rho_{2}$. Однако соотношение $\Phi\left[\rho_{1}\right]=\Phi\left[\rho_{2}\right]$ эквивалентно тому, что $\operatorname{Tr} \rho_{1} \Phi^{*}\left[D_{B}(\mathbf{z})\right]=\operatorname{Tr} \rho_{2} \Phi^{*}\left[D_{B}(\mathbf{z})\right]$, т. е. $\operatorname{Tr} \rho_{1} D_{A}(\mathbf{K z})=\operatorname{Tr} \rho_{2} D_{A}(\mathbf{K z})$ для всех $\mathbf{z} \in \mathbf{Z}_{B}$. В силу неприводимости системы Вейля это свойство эквивалентно тому, что $\operatorname{Ran} \mathbf{K}=\mathbf{Z}_{A}$, т. е. $\operatorname{Ker} \mathbf{K}^{*}=\{0\}$ или же $\mathbf{K K}^{*}>0$. Лемма доказана.

Канал (49) является экстремальным, если $\boldsymbol{\mu}$ - минимальное решение неравенства (50). Специальные случаи отображений (49) даются аттенюатором и усилителем, которые характеризуются матрицей $\mathbf{K}$, удовлетворяющей условиям $\mathbf{K}^{*} \mathbf{K} \leqslant \mathbf{I}_{B}$ и $\mathbf{K}^{*} \mathbf{K} \geqslant \mathbf{I}_{B}$ соответственно. Нас особенно интересует экстремальный аттенюатор, который отвечает параметрам

$$
\mathbf{K}^{*} \mathbf{K} \leqslant \mathbf{I}_{B}, \quad \boldsymbol{\mu}=\frac{1}{2}\left(\mathbf{I}_{B}-\mathbf{K}^{*} \mathbf{K}\right),
$$

и экстремальный усилитель с

$$
\mathbf{K}^{*} \mathbf{K} \geqslant \mathbf{I}_{B}, \quad \boldsymbol{\mu}=\frac{1}{2}\left(\mathbf{K}^{*} \mathbf{K}-\mathbf{I}_{B}\right)
$$

Гауссовский калибровочно-контравариантный канал (46) действует по формуле

$$
\Phi^{*}\left[D_{B}(\mathbf{z})\right]=D_{A}(-\overline{\mathbf{K} z}) \exp \left\{-\mathbf{z}^{*} \boldsymbol{\mu} \mathbf{z}\right\}
$$

где $\boldsymbol{\mu}$ - эрмитова матрица, удовлетворяющая соотношению

$$
\boldsymbol{\mu} \geqslant \frac{1}{2}\left(\mathbf{I}_{B}+\mathbf{K}^{*} \mathbf{K}\right)
$$

которое следует из (47). Здесь $\overline{\mathbf{z}}-$ вектор-столбец, состоящий из комплексно-сопряженных компонент вектора z. Эти отображения экстремальны, если

$$
\boldsymbol{\mu}=\frac{1}{2}\left(\mathbf{I}_{B}+\mathbf{K}^{*} \mathbf{K}\right)
$$

Следующее предложение дает обобщение на многомодовый случай полезного и важного разложения одномодовых каналов (см. [20], [13]).

ПрЕДЛОЖЕНИЕ 9. Произволъный гауссовский калибровочно-ковариантный канал $\Phi: A \rightarrow B$ является композицией $\Phi=\Phi_{2} \circ \Phi_{1}$ экстремального аттенюатора $\Phi_{1}: A \rightarrow B$ и экстремального усилителя $\Phi_{2}: B \rightarrow B$.

Произвольный гауссовский калибровочно-контравариантный канал $\Phi: A \rightarrow$ $B$ является композииией экстремального аттенюатора $\Phi_{1}: A \rightarrow B$ и экстремального калибровочно-контравариантного канала $\Phi_{2}: B \rightarrow B$.

${ }^{8}$ Для эрмитовых матриц $M, N$ строгое неравенство $M>N$ означает, что матрица $M-N$ положительно определена. 
ДокАЗАТЕЛЬСтво. Композиция $\Phi=\Phi_{2} \circ \Phi_{1}$ гауссовских калибровочно-ковариантных каналов $\Phi_{1}$ и $\Phi_{2}$ подчиняется правилу

$$
\begin{aligned}
\mathbf{K} & =\mathbf{K}_{1} \mathbf{K}_{2}, \\
\boldsymbol{\mu} & =\mathbf{K}_{2}^{*} \boldsymbol{\mu}_{1} \mathbf{K}_{2}+\boldsymbol{\mu}_{2} .
\end{aligned}
$$

Подставляя соотношения

$$
\begin{aligned}
& \boldsymbol{\mu}_{1}=\frac{1}{2}\left(\mathbf{I}_{B}-\mathbf{K}_{1}^{*} \mathbf{K}_{1}\right)=\frac{1}{2}\left(\mathbf{I}_{B}-|\mathbf{K}|_{1}^{2}\right), \\
& \boldsymbol{\mu}_{2}=\frac{1}{2}\left(\mathbf{K}_{2}^{*} \mathbf{K}_{2}-\mathbf{I}_{B}\right)=\frac{1}{2}\left(\left|\mathbf{K}_{2}\right|^{2}-\mathbf{I}_{B}\right)
\end{aligned}
$$

в (57) и используя (56), получаем

$$
\left|\mathbf{K}_{2}\right|^{2}=\mathbf{K}_{2}^{*} \mathbf{K}_{2}=\boldsymbol{\mu}+\frac{1}{2}\left(\mathbf{K}^{*} \mathbf{K}+\mathbf{I}_{B}\right) \geqslant\left\{\begin{array}{l}
\mathbf{I}_{B} \\
\mathbf{K}^{*} \mathbf{K}
\end{array}\right.
$$

из неравенства (50). Используя операторную монотонность квадратного корня, имеем

$$
\left|\mathbf{K}_{2}\right| \geqslant \mathbf{I}_{B}, \quad\left|\mathbf{K}_{2}\right| \geqslant|\mathbf{K}| .
$$

Из первого неравенства (58) следует, что, выбирая

$$
\mathbf{K}_{2}=\left|\mathbf{K}_{2}\right|=\sqrt{\boldsymbol{\mu}+\frac{1}{2}\left(\mathbf{K}^{*} \mathbf{K}+\mathbf{I}_{B}\right)}
$$

и соответствующую матрицу $\boldsymbol{\mu}_{2}=\left(\left|\mathbf{K}_{2}\right|^{2}-\mathbf{I}_{B}\right) / 2$, мы получаем экстремальный усилитель $\Phi_{2}: B \rightarrow B$.

Тогда, полагая

$$
\mathbf{K}_{1}=\mathbf{K}\left|\mathbf{K}_{2}\right|^{-1}
$$

и принимая во внимание второе неравенство в (58) и лемму 10 ниже, мы получаем

$$
\mathbf{K}_{1} \mathbf{K}_{1}^{*}=\mathbf{K}\left|\mathbf{K}_{2}\right|^{-2} \mathbf{K}^{*}=\mathbf{K}\left[\boldsymbol{\mu}+\frac{1}{2}\left(\mathbf{K}^{*} \mathbf{K}+\mathbf{I}\right)\right]^{-1} \mathbf{K}^{*} \leqslant \mathbf{I}_{A},
$$

откуда следует неравенство $\mathbf{K}_{1}^{*} \mathbf{K}_{1} \leqslant \mathbf{I}_{A}$, так что $\mathbf{K}_{1}$ вместе с соответствующей матрицей $\boldsymbol{\mu}_{1}=\left(\mathbf{I}_{B}-\mathbf{K}_{1}^{*} \mathbf{K}_{1}\right) / 2$ дают экстремальный аттенюатор.

Лемма 10. Пусть $\mathbf{M} \geqslant \mathbf{K}^{*} \mathbf{K}$, тогда $\mathbf{K M}^{-} \mathbf{K}^{*} \leqslant \mathbf{I}_{A}$, где ${ }^{-}$означает (обобщенную) обратную матрицу.

ДокАЗАТЕльство. Согласно определению обобщенной обратной матрицы,

$$
u^{*} \mathbf{M}^{-} u=\sup _{v: v \in \operatorname{Ran} \mathbf{M}, v \neq 0} \frac{\left|u^{*} v\right|^{2}}{v^{*} \mathbf{M} v} .
$$

Подставляя $\mathbf{K}^{*} u$ вместо $u$ и используя неравенство Коши-Буняковского в числителе дроби, получаем

$$
u^{*} \mathbf{K M}^{-} \mathbf{K}^{*} u \leqslant \sup _{v: v \in \operatorname{Ran} \mathbf{M}, v \neq 0} \frac{u^{*} u v^{*} \mathbf{K}^{*} \mathbf{K} v}{v^{*} \mathbf{M} v} \leqslant u^{*} u .
$$

Лемма доказана. 
В случае контравариантного канала соотношения (56), (57) заменяются на

$$
\begin{aligned}
\overline{\mathbf{K}} & =\mathbf{K}_{1} \overline{\mathbf{K}}_{2}, \\
\boldsymbol{\mu} & =\mathbf{K}_{2}^{*} \overline{\boldsymbol{\mu}}_{1} \mathbf{K}_{2}+\boldsymbol{\mu}_{2} .
\end{aligned}
$$

Подставляя

$$
\boldsymbol{\mu}_{1}=\frac{1}{2}\left(\mathbf{I}-\mathbf{K}_{1}^{*} \mathbf{K}_{1}\right), \quad \boldsymbol{\mu}_{2}=\frac{1}{2}\left(\mathbf{K}_{2}^{*} \mathbf{K}_{2}+\mathbf{I}_{B}\right)
$$

в (63) и используя (54), получаем

$$
\left|\mathbf{K}_{2}\right|^{2}=\mathbf{K}_{2}^{*} \mathbf{K}_{2}=\boldsymbol{\mu}+\frac{1}{2}\left(\mathbf{K}^{*} \mathbf{K}-\mathbf{I}_{B}\right) \geqslant \mathbf{K}^{*} \mathbf{K} .
$$

Полагая $\mathbf{K}_{2}=\left|\mathbf{K}_{2}\right|, \boldsymbol{\mu}_{2}=\left(\left|\mathbf{K}_{2}\right|^{2}+\mathbf{I}_{B}\right) / 2$, получаем экстремальный калибровочно-контравариантный канал $\Phi_{2}: B \rightarrow B$. Полагая

$$
\overline{\mathbf{K}}_{1}=\mathbf{K}\left|\mathbf{K}_{2}\right|^{-}
$$

и используя лемму 10, мы получаем

$$
\begin{aligned}
\overline{\mathbf{K}}_{1} \overline{\mathbf{K}}_{1}^{*} & =\mathbf{K}\left(\left|\mathbf{K}_{2}\right|^{-}\right)^{2} \mathbf{K}^{*} \\
& =\mathbf{K}\left[\boldsymbol{\mu}+\frac{1}{2}\left(\mathbf{K}^{*} \mathbf{K}-\mathbf{I}_{B}\right)\right]^{-} \mathbf{K}^{*} \leqslant \mathbf{I}_{A},
\end{aligned}
$$

откуда следует неравенство $\mathbf{K}_{1} \mathbf{K}_{1}^{*} \leqslant \mathbf{I}_{A}$, поэтому $\mathbf{K}_{1}$ с соответствующей матрицей $\boldsymbol{\mu}_{1}$ дают экстремальный аттенюатор $\Phi_{1}: A \rightarrow B$. Предложение 9 доказано.

ЗАмЕчАниЕ 11. В случае калибровочно-ковариантного канала соотношение (61) показывает, что из неравенства $\mathbf{K} \mathbf{K}^{*}>0$ следует $\mathbf{K}_{1} \mathbf{K}_{1}^{*}>0$, а неравенство $\boldsymbol{\mu}>\left(\mathbf{K}^{*} \mathbf{K}-\mathbf{I}_{B}\right) / 2$ влечет за собой $\mathbf{K}_{1} \mathbf{K}_{1}^{*}<\mathbf{I}_{A}$. В случае калибровочно-контравариантного канала неравенство $\boldsymbol{\mu}>\left(\mathbf{I}_{B}+\mathbf{K}^{*} \mathbf{K}\right) / 2$ влечет за собой $0<\mathbf{K}_{1} \mathbf{K}_{1}^{*}<\mathbf{I}_{A}$ в силу (66).

ПРЕДЛОЖЕНИЕ 12. Экстремальный аттенюатор с матрицей $\mathbf{K}$ и экстремальный аттенюатор с матрицей $\widetilde{\mathbf{K}}=\sqrt{\mathbf{I}_{A}-\mathbf{K K}^{*}}$ взаимно комплементарны.

Экстремальный усилитель с матрицей $\mathbf{K}$ и экстремальный калибровочно-контравариантный канал с матрицей $\widetilde{\mathbf{K}}=\sqrt{\overline{\mathbf{K}} \overline{\mathbf{K}}^{*}-\mathbf{I}_{A}}$ взаимно комплементарны.

ДоКАЗАТЕЛЬСтво. Случай одной моды см. в [13] или [38; п. 12.6.1]. В многомодовом случае положим по определению $\mathbf{Z}_{E} \simeq \mathbf{Z}_{A}, \mathbf{Z}_{D} \simeq \mathbf{Z}_{B}$, так что $\mathbf{Z}=\mathbf{Z}_{A} \oplus \mathbf{Z}_{D} \simeq \mathbf{Z}_{B} \oplus \mathbf{Z}_{E}$

В случае аттенюатора рассмотрим блочный унитарный оператор в $\mathbf{Z}$ :

$$
\mathbf{V}=\left[\begin{array}{cc}
\mathbf{K} & \sqrt{\mathbf{I}_{A}-\mathbf{K K}^{*}} \\
\sqrt{\mathbf{I}_{B}-\mathbf{K}^{*} \mathbf{K}} & -\mathbf{K}^{*}
\end{array}\right],
$$

который определяет унитарную динамику $U$ в $\mathscr{H}=\mathscr{H}_{A} \otimes \mathscr{H}_{D} \simeq \mathscr{H}_{B} \otimes \mathscr{H}_{E}$

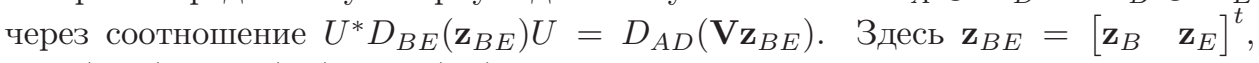
$D_{B E}\left(\mathbf{z}_{B E}\right)=D_{B}\left(\mathbf{z}_{B}\right) \otimes D_{E}\left(\mathbf{z}_{E}\right)$ и унитарность следует из соотношения

$$
\mathbf{K} \sqrt{\mathbf{I}_{B}-\mathbf{K}^{*} \mathbf{K}}=\sqrt{\mathbf{I}_{A}-\mathbf{K K}^{*}} \mathbf{K} .
$$


Пусть $\rho_{D}=\rho_{0}$ - вакуумное состояние, $\rho_{A}=\rho$ - произвольное состояние. Тогда формулы (7), (8) определяют взаимно комплементарные экстремальные аттенюаторы, что и составляет первое утверждение. Доказательство получается вычислением характеристической функции выходного состояния каналов. Для состояния составной системы $\rho_{B E}=U\left(\rho \otimes \rho_{D}\right) U^{*}$ имеем

$$
\begin{aligned}
\phi_{B E}\left(\mathbf{z}_{B E}\right) & =\operatorname{Tr} U\left(\rho \otimes \rho_{D}\right) U^{*}\left[D_{B}\left(\mathbf{z}_{B}\right) \otimes D_{E}\left(\mathbf{z}_{E}\right)\right] \\
& =\operatorname{Tr}\left(\rho \otimes \rho_{D}\right) U^{*}\left[D_{B}\left(\mathbf{z}_{B}\right) \otimes D_{E}\left(\mathbf{z}_{E}\right)\right] U \\
& =\operatorname{Tr}\left(\rho \otimes \rho_{D}\right)\left[D_{A}\left(\mathbf{K} \mathbf{z}_{B}+\widetilde{\mathbf{K}} \mathbf{z}_{E}\right) \otimes D_{D}\left(\sqrt{\mathbf{I}_{B}-\mathbf{K}^{*} \mathbf{K}} \mathbf{z}_{B}-\mathbf{K}^{*} \mathbf{z}_{E}\right)\right] \\
& =\phi_{A}\left(\mathbf{K} \mathbf{z}_{B}+\widetilde{\mathbf{K}} \mathbf{z}_{E}\right) \exp \left\{-\frac{1}{2}\left|\sqrt{\mathbf{I}_{B}-\mathbf{K}^{*} \mathbf{K}} \mathbf{z}_{B}-\mathbf{K}^{*} \mathbf{z}_{E}\right|^{2}\right\} .
\end{aligned}
$$

Полагая $\mathbf{z}_{E}=0$ или $\mathbf{z}_{B}=0$, мы получаем

$$
\begin{aligned}
& \phi_{B}\left(\mathbf{z}_{B}\right)=\phi_{A}\left(\mathbf{K} \mathbf{z}_{B}\right) \exp \left\{-\frac{1}{2} \mathbf{z}_{B}^{*}\left(\mathbf{I}_{B}-\mathbf{K}^{*} \mathbf{K}\right) \mathbf{z}_{B}\right\}, \\
& \phi_{E}\left(\mathbf{z}_{E}\right)=\phi_{A}\left(\widetilde{\mathbf{K}} \mathbf{z}_{E}\right) \exp \left\{-\frac{1}{2} \mathbf{z}_{E}^{*} \mathbf{K} \mathbf{K}^{*} \mathbf{z}_{E}\right\},
\end{aligned}
$$

что и требовалось.

В случае усилителя рассмотрим блочный оператор

$$
\mathbf{V}=\left[\begin{array}{cc}
\mathbf{K} & -\sqrt{\mathbf{K} \mathbf{K}^{*}-\mathbf{I}_{A}} \Lambda \\
-\Lambda \sqrt{\mathbf{K}^{*} \mathbf{K}-\mathbf{I}_{B}} & \Lambda \mathbf{K}^{*} \Lambda
\end{array}\right],
$$

где $\Lambda$ - оператор комплексного сопряжения, антикоммутирующий с умножением на $i$. Используя тот факт, что $\Delta\left(\Lambda z, \Lambda z^{\prime}\right)=-\Delta\left(z, z^{\prime}\right)$, получаем, что $\mathbf{V}$ отвечает симплектическому преобразованию в $\mathbf{Z}$, порождающему унитарную динамику $U$ в $\mathscr{H}$. Пусть вновь $\rho_{0}$ - вакуумное состояние окружения. Тогда формулы (7), (8) определяют взаимно комплементарные каналы из второго утверждения предложения, причем доказательство аналогично первому. Предложение доказано.

В дальнейшем нам понадобится также следующая лемма.

Лемма 13. Пусть $\Phi_{1}: A \rightarrow B$ - экстремальный аттенюатор такой, что $\mathbf{0}<\mathbf{K}_{1} \mathbf{K}_{1}^{*}<\mathbf{I}_{A}$. $B$ этом случае $\Phi_{1}\left[P_{\psi}\right]=P_{\psi^{\prime}}$ (чистое состояние) тогда и только тогда, когда $P_{\psi}$ - когерентное состояние.

ДокАЗАТЕльство. Согласно предложению 12 , комплементарный канал $\widetilde{\Phi}_{1}$ является экстремальным аттенюатором с матрицей $\widetilde{\mathbf{K}}=\sqrt{\mathbf{I}_{A}-\mathbf{K}_{1} \mathbf{K}_{1}^{*}}$, так что $0<\widetilde{\mathbf{K}}<\mathbf{I}_{A}$. Его выходное состояние также является чистым, $\Phi_{1}\left[P_{\psi}\right]=P_{\psi_{E}^{\prime}}$, поскольку выходные состояния комплементарных каналов имеют одинаковые ненулевые спектры согласно лемме 3. Таким образом,

$$
U\left(\psi \otimes \psi_{0}\right)=\psi^{\prime} \otimes \psi_{E}^{\prime},
$$

где $\psi_{0} \in \mathscr{H}_{D}$ - вакуумный вектор, а $U$ - унитарный оператор в $\mathscr{H}$, реализующий симплектическое преобразование, соответствующее унитарному оператору (67) в $\mathbf{Z}_{A} \oplus \mathbf{Z}_{D} \simeq \mathbf{Z}_{B} \oplus \mathbf{Z}_{E}$, где $\mathbf{Z}_{D} \simeq \mathbf{Z}_{B}, \mathbf{Z}_{E} \simeq \mathbf{Z}_{A}$. Обозначая

$$
\phi(\mathbf{z})=\operatorname{Tr} P_{\psi} D_{A}(\mathbf{z}), \quad \phi^{\prime}\left(\mathbf{z}_{B}\right)=\operatorname{Tr} P_{\psi^{\prime}} D_{B}\left(\mathbf{z}_{B}\right), \quad \phi_{E}\left(\mathbf{z}_{E}\right)=\operatorname{Tr} P_{\psi_{E}^{\prime}} D_{E}\left(\mathbf{z}_{E}\right)
$$


квантовые характеристические функции и используя соотношение (69), получаем функциональное уравнение

$$
\phi^{\prime}\left(\mathbf{z}_{B}\right) \phi_{E}\left(\mathbf{z}_{E}\right)=\phi\left(\mathbf{K}_{1} \mathbf{z}_{B}+\widetilde{\mathbf{K}} \mathbf{z}_{E}\right) \exp \left\{-\frac{1}{2}\left|\sqrt{\mathbf{I}_{B}-\mathbf{K}_{1}^{*} \mathbf{K}_{1}} \mathbf{z}_{B}-\mathbf{K}_{1}^{*} \mathbf{z}_{E}\right|^{2}\right\} .
$$

Полагая $\mathbf{z}_{E}=0$, соответственно $\mathbf{z}=0$, получаем

$$
\begin{aligned}
\phi^{\prime}\left(\mathbf{z}_{B}\right) & =\phi\left(\mathbf{K}_{1} \mathbf{z}_{B}\right) \exp \left\{-\frac{1}{2}\left|\sqrt{\mathbf{I}_{B}-\mathbf{K}_{1}^{*} \mathbf{K}_{1}} \mathbf{z}_{B}\right|^{2}\right\}, \\
\phi_{E}\left(\mathbf{z}_{E}\right) & =\phi\left(\widetilde{\mathbf{K}} \mathbf{z}_{E}\right) \exp \left\{-\frac{1}{2}\left|\mathbf{K}_{1}^{*} \mathbf{z}_{E}\right|^{2}\right\}
\end{aligned}
$$

таким образом, после замены переменных $\mathbf{z}=\mathbf{K}_{1} \mathbf{z}_{B}, \mathbf{z}^{\prime}=\widetilde{\mathbf{K}} \mathbf{z}_{E}$ и с учетом соотношения (68) уравнение (70) сводится к

$$
\phi(\mathbf{z}) \phi\left(\mathbf{z}^{\prime}\right)=\phi\left(\mathbf{z}+\mathbf{z}^{\prime}\right) \exp \left\{\operatorname{Re} \mathbf{z}^{*} \mathbf{z}^{\prime}\right\}
$$

Условие леммы гарантирует, что $\operatorname{Ran} \mathbf{K}_{1}=\operatorname{Ran} \widetilde{\mathbf{K}}=\mathbf{Z}_{A}$. Подставляя $\omega(\mathbf{z})=$ $\phi(\mathbf{z}) \exp \left\{|\mathbf{z}|^{2} / 2\right\}$, получаем

$$
\omega(\mathbf{z}) \omega\left(\mathbf{z}^{\prime}\right)=\omega\left(\mathbf{z}+\mathbf{z}^{\prime}\right)
$$

для всех $\mathbf{z}, \mathbf{z}^{\prime} \in \mathbf{Z}_{A}$. Функция $\omega(\mathbf{z})$, как и характеристическая функция $\phi(\mathbf{z})$, непрерывна и удовлетворяет условию $\omega(-\mathbf{z})=\overline{\omega(\mathbf{z})}$. Единственным решением уравнения (71), удовлетворяющим этим условиям, является экспонента $\omega(\mathbf{z})=$ $\exp \left\{i \operatorname{Im} \mathbf{w}^{*} \mathbf{z}\right\}$ для некоторого комплексного $\mathbf{w}$. Таким образом, функция

$$
\phi(\mathbf{z})=\exp \left\{i \operatorname{Im} \mathbf{w}^{*} \mathbf{z}-\frac{1}{2}|\mathbf{z}|^{2}\right\}
$$

является характеристической функцией когерентного состояния $\rho_{\mathbf{w} / 2}$. Лемма доказана.

3.4. Гауссовские оптимизаторы. Следующий основной результат был получен в работе [53] для случая одной моды. Здесь мы приводим полное доказательство в многомодовом случае, набросок которого дан в [23].

Теорема 14. (i) Пусть $\Phi$ - гауссовский калибровочно-ковариантный или контравариантный канал, и пусть $f$ - вещественная вогнутая функиия на $[0,1]$ такая, что $f(0)=0$, тогда

$$
\operatorname{Tr} f(\Phi[\rho]) \geqslant \operatorname{Tr} f\left(\Phi\left[\rho_{\mathbf{w}}\right]\right)=\operatorname{Tr} f\left(\Phi\left[\rho_{0}\right]\right)
$$

для всех состояний $\rho$ и любого когерентного состояния $\rho_{\mathbf{w}}$ (значение в правой части одно и то же для всех когерентных состояний в силу свойства унитарной ковариантности гауссовского канала (34)). 
(ii) Пусть функиия $f$ строго вогнута, тогда неравенство в (72) превращается в равенство, толъко если $\rho$ - когерентное состояние и выполнено одно из следующих условий:

(а) $s_{A}=s_{B}$ и канал $\Phi$ является экстремальным усилителем $c$

$$
\boldsymbol{\mu}=\frac{1}{2}\left(\mathbf{K}^{*} \mathbf{K}-\mathbf{I}_{B}\right)>0 ;
$$

(b) $s_{B} \geqslant s_{A}$, канал $\Phi$ является калибровочно-ковариантным с $\mathbf{K K}^{*}>0$ и

$$
\boldsymbol{\mu}>\frac{1}{2}\left(\mathbf{K}^{*} \mathbf{K}-\mathbf{I}_{B}\right) ;
$$

(c) $s_{B} \geqslant s_{A}$, канал $\Phi$ является калибровочно-контравариантным с $\mathbf{K K}^{*}>0$ u

$$
\boldsymbol{\mu}>\frac{\mathbf{1}}{\mathbf{2}}\left(\mathbf{I}_{B}+\mathbf{K}^{*} \mathbf{K}\right) .
$$

ДокАзАтельство. (i) Сначала докажем первое утверждение для строго вогнутых функций $f$. В этом случае неравенство (72) для произвольной вогнутой $f$ получается путем монотонной аппроксимации $f(x)=\lim _{\varepsilon \downarrow 0} f_{\varepsilon}(x)$ строго вогнутыми функциями $f_{\varepsilon}(x)=f(x)-\varepsilon x^{2}$. Также, в силу вогнутости, достаточно доказать (72) только для $\rho=P_{\psi}$.

В силу предложения 9 имеем $\Phi=\Phi_{2} \circ \Phi_{1}$, где $\Phi_{1}$ - экстремальный аттенюатор и $\Phi_{2}: B \rightarrow B$ - либо экстремальный усилитель, либо экстремальный калибровочно-контравариантный канал. Любой экстремальный аттенюатор отображает вакуумное состояние в себя. В самом деле,

$$
\begin{aligned}
\operatorname{Tr} \Phi_{1}\left[\rho_{0}\right] D_{B}(\mathbf{z}) & =\operatorname{Tr} \rho_{0} \Phi_{1}^{*}\left[D_{B}(\mathbf{z})\right] \\
& =\operatorname{Tr} \rho_{0} D_{A}(\mathbf{K} \mathbf{z}) \exp \left\{-\frac{1}{2} \mathbf{z}^{*}\left(\mathbf{I}_{B}-\mathbf{K}^{*} \mathbf{K}\right) \mathbf{z}\right\} \\
& =\exp \left\{-\frac{1}{2}|\mathbf{z}|^{2}\right\}=\operatorname{Tr} \rho_{0} D_{B}(\mathbf{z}) .
\end{aligned}
$$

Поэтому $\operatorname{Tr} f\left(\Phi\left[\rho_{0}\right]\right)=\operatorname{Tr} f\left(\Phi_{2}\left[\rho_{0}\right]\right)$. Тогда достаточно доказать $(72)$ для всех экстремальных усилителей и для всех экстремальных калибровочно-контравариантных каналов $\Phi_{2}$. В самом деле, предположим, что мы доказали неравенство

$$
\operatorname{Tr} f\left(\Phi_{2}\left[P_{\psi}\right]\right) \geqslant \operatorname{Tr} f\left(\Phi_{2}\left[\rho_{0}\right]\right)
$$

для любого вектора состояния $\psi$. Рассмотрим спектральное разложение

$$
\Phi_{1}\left[P_{\psi}\right]=\sum_{j} p_{j} P_{\phi_{j}}, \quad \text { где } p_{j}>0,
$$

тогда

$$
\begin{aligned}
\operatorname{Tr} f\left(\Phi\left[P_{\psi}\right]\right) & =\operatorname{Tr} f\left(\Phi_{2}\left[\Phi_{1}\left[P_{\psi}\right]\right]\right) \\
& \geqslant \sum_{j} p_{j} \operatorname{Tr} f\left(\Phi_{2}\left[P_{\phi_{j}}\right]\right) \\
& \geqslant \operatorname{Tr} f\left(\Phi_{2}\left[\rho_{0}\right]\right) \\
& =\operatorname{Tr} f\left(\Phi_{2}\left[\Phi_{1}\left[\rho_{0}\right]\right]\right)=\operatorname{Tr} f\left(\Phi\left[\rho_{0}\right]\right) .
\end{aligned}
$$


Согласно второму утверждению предложения 12 и лемме 3

$$
\operatorname{Tr} f\left(\Phi_{2}\left[P_{\psi}\right]\right)=\operatorname{Tr} f\left(\widetilde{\Phi}_{2}\left[P_{\psi}\right]\right),
$$

где $\Phi_{2}$ - экстремальный усилитель, а $\widetilde{\Phi}_{2}$ - экстремальный калибровочно-контравариантный канал. Поэтому достаточно доказать соотношение (74) только для экстремального усилителя $\Phi_{2}: B \rightarrow B$ с эрмитовой матрицей $\mathbf{K}_{2} \geqslant \mathbf{I}_{B}$.

В основе следующего результата лежит ключевое наблюдение Джованнетти.

Лемма 15. Для экстремального усилителя $\Phi_{2}: B \rightarrow B$ с матрицей $\mathbf{K}_{2} \geqslant \mathbf{I}_{B}$ существует экстремальный аттенюатор $\Phi_{1}^{\prime}$ такой, что для всех $\psi \in \mathscr{H}_{B}$

$$
\Phi_{2}\left(P_{\psi}\right) \sim\left(\Phi_{2} \circ \Phi_{1}^{\prime}\right)\left(P_{\psi}\right) .
$$

ДокАЗАтельство. Согласно предложению 12 и лемме $3, \Phi_{2}\left(P_{\psi}\right) \sim \widetilde{\Phi}_{2}\left(P_{\psi}\right)$ для всех $\psi \in \mathscr{H}_{B}$, где $\widetilde{\Phi}_{2}$ - экстремальный контравариантный канал с матрицей $\widetilde{\mathbf{K}}=\sqrt{\overline{\mathbf{K}}_{2}^{2}-\mathbf{I}_{B}}$.

Зададим отображение транспонирования $\mathscr{T}: B \rightarrow B$ соотношением

$$
\mathscr{T}[D(\mathbf{z})]=D(-\overline{\mathbf{z}})
$$

Композиция $\Phi=\mathscr{T} \circ \widetilde{\Phi}_{2}$ является ковариантным гауссовским каналом:

$$
\Phi^{*}[D(\mathbf{z})]=\widetilde{\Phi}_{2}^{*} \circ \mathscr{T}[D(\mathbf{z})]=D\left(\sqrt{\mathbf{K}_{2}^{2}-\mathbf{I}_{B}} \mathbf{z}\right) \exp \left\{-\frac{1}{2} \mathbf{z}^{*} \mathbf{K}_{2}^{2} \mathbf{z}\right\} .
$$

Применяя разложение из предложения 9 , получаем $\Phi=\Phi_{2} \circ \Phi_{1}^{\prime}$, где $\Phi_{2}-$ исходный усилитель, а $\Phi_{1}^{\prime}: B \rightarrow B$ - другой экстремальный аттенюатор с матрицей $\mathbf{K}_{1}=\sqrt{\mathbf{I}_{B}-\mathbf{K}_{2}^{-2}}$. Отсюда следует соотношение (79). Лемма доказана.

Леммы 15 и 5 влекут равенство

$$
\operatorname{Tr} f\left(\Phi_{2}\left(P_{\psi}\right)\right)=\operatorname{Tr} f\left(\left(\Phi_{2} \circ \Phi_{1}^{\prime}\right)\left(P_{\psi}\right)\right) .
$$

Вновь рассмотрим спектральное разложение оператора плотности

$$
\Phi_{1}^{\prime}\left(P_{\psi}\right)=\sum_{j} p_{j}^{\prime} P_{\psi_{j}}, \quad p_{j}^{\prime}>0
$$

В силу вогнутости имеем

$$
\operatorname{Tr} f\left(\left(\Phi_{2} \circ \Phi_{1}^{\prime}\right)\left[P_{\psi}\right]\right) \geqslant \sum_{j} p_{j}^{\prime} \operatorname{Tr} f\left(\Phi_{2}\left[P_{\psi_{j}}\right]\right) .
$$

Поскольку функция $f$ по предположению строго вогнута, то функционал $\rho \rightarrow \operatorname{Tr} f\left(\Phi_{2}[\rho]\right)$ строго вогнут [11]. Предположив, что $P_{\psi}$ является минимизатором для функционала $(80)$, мы получаем, что все операторы $\Phi_{2}\left[P_{\psi_{j}}\right]$ должны совпадать, поскольку в противном случае неравенство (81) было бы строгим, что противоречит предположению. Из леммы 8 следует, что $P_{\psi_{j}}=P_{\psi^{\prime}}$ для всех $j$ и некоторого $\psi^{\prime} \in \mathscr{H}_{B}$, поэтому, в предположении, что $P_{\psi}$ является минимизатором, выходное состояние $\Phi_{1}\left[P_{\psi}\right]=P_{\psi^{\prime}}$ является чистым. 
Поскольку $\mathbf{K}_{1}=\sqrt{\mathbf{I}_{B}-\mathbf{K}_{2}^{-2}}$, условие леммы 13 выполнено, если $\mathbf{K}_{2}>\mathbf{I}_{B}$. В этом случае, если $P_{\psi}$ является минимизатором, то из леммы 13 следует, что $P_{\psi}$ - когерентное состояние. Таким образом, мы получаем неравенство (74) для усилителя $\Phi_{2}$ с $\mathbf{K}_{2}>\mathbf{I}_{B}$ в случае строго вогнутой функции $f$. Отсюда также следует случай а) утверждения (ii).

В случае усилителя $\Phi_{2}$ с матрицей $\mathbf{K}_{2} \geqslant \mathbf{I}_{B}$ мы можем рассмотреть любую последовательность $\mathbf{K}_{2}^{(n)}>\mathbf{I}_{B}, \mathbf{K}_{2}^{(n)} \rightarrow \mathbf{K}_{2}$, и соответствующие усилители $\Phi_{2}^{(n)}$. Тогда $\operatorname{Tr} f\left(\Phi_{2}^{(n)}[\rho]\right) \rightarrow \operatorname{Tr} f\left(\Phi_{2}[\rho]\right)$ для любой вогнутой полигональной функции $f$ на $[0,1]$ такой, что $f(0)=0$, и любого оператора плотности $\rho \in \mathfrak{S}\left(\mathscr{H}_{A}\right)$. Это следует из того, что всякая такая функция удовлетворяет условию Липшица, $|f(x)-f(y)| \leqslant \varkappa|x-y|$, и $\left\|\Phi_{2}^{(n)}[\rho]-\Phi_{2}[\rho]\right\|_{1} \rightarrow 0$. Отсюда следует, что (74) выполняется для всех экстремальных усилителей $\Phi_{2}$ в случае вогнутой полигональной функции $f$. Для произвольной вогнутой $f$ на $[0,1]$ найдется монотонно неубывающая последовательность вогнутых полигональных функций $f_{m}$, сходящаяся поточечно к $f$. Переходя к пределу при $m \rightarrow \infty$, получаем неравенство (74) для произвольного экстремального усилителя, и поэтому неравенство (72) имеет место для любого гауссовского калибровочно-ковариантного или контравариантного канала.

(ii) Утверждение "только если" в случаях b), с) получается из разложения $\Phi=\Phi_{2} \circ \Phi_{1}$ и соотношений (75)-(77) путем рассуждений, аналогичных случаю экстремального усилителя. Заметим, что из условий, наложенных на канал $\Phi$, следует, что в разложении $\Phi=\Phi_{2} \circ \Phi_{1}$ аттенюатор $\Phi_{1}$ определяется матрицей $\mathbf{K}_{1}$ такой, что $0<\mathbf{K}_{1} \mathbf{K}_{1}^{*}<\mathbf{I}_{A}$ (см. замечание 11 ). Применяя рассуждение, относящееся к соотношениям $(80)$ и (81) со строго вогнутой $f$, к соотношениям (75)-(78), мы получаем, что для любого чистого минимизатора $P_{\psi}$ функционала $\operatorname{Tr} f\left(\Phi\left[P_{\psi}\right]\right)$ выход экстремального аттенюатора $\Phi_{1}\left[P_{\psi}\right]$ обязательно является чистым состоянием. Применяя лемму 13 к аттенюатору $\Phi_{1}$, мы заключаем, что $P_{\psi}$ с необходимостью является когерентным состоянием.

Теорема 14 доказана.

\section{5. Явные формулы и аддитивность.}

ПРеДЛОЖеНИЕ 16. Для любого $p>1$ и любого гауссовского калибровочно-ковариантного или контравариантного канала $\Phi$

$$
\begin{aligned}
\|\Phi\|_{1 \rightarrow p} & =\left(\operatorname{Tr} \Phi\left[\rho_{0}\right]^{p}\right)^{1 / p} \\
\check{R}_{p}(\Phi) & =R_{p}\left(\Phi\left[\rho_{0}\right]\right) \\
\check{H}(\Phi) & =H\left(\Phi\left[\rho_{0}\right]\right),
\end{aligned}
$$

где $\rho_{0}$ - вакуумное состояние.

Свойство мультипликативности (14) выполняется для любых двух гауссовских калибровочно-ковариантных (контравариантных) каналов $\Phi_{1} u \Phi_{2}$, как и свойство аддитивности для минимальной энтропии Ренъи (15) и минимальной энтропии фон Неймана (16).

ДокАзАтЕльство. Первое утверждение следует из теоремы 14, если положить $f(x)=-x^{p}, f(x)=-x \log x$. 
Если $\Phi_{1}$ и $\Phi_{2}$ оба калибровочно ковариантны (контравариантны), то их тензорное произведение $\Phi_{1} \otimes \Phi_{2}$ также имеет это свойство. Второе утверждение тогда следует из (82)-(84) и свойства мультипликативности вакуумного состояния $\rho_{0}=\rho_{0}^{(1)} \otimes \rho_{0}^{(2)}$, вытекающего из определения. Предложение доказано.

Из определений калибровочно-ковариантных (контравариантных) каналов (49), (53) вытекает, что состояние $\Phi\left[\rho_{0}\right]$ является гауссовским калибровочно-инвариантным с корреляционной матрицей $\boldsymbol{\mu}+\mathbf{K}^{*} \mathbf{K} / 2$. Спектр оператора $\Phi\left[\rho_{0}\right]$ вычисляется в явном виде, что приводит к выражениям (см. [41])

$$
\|\Phi\|_{1 \rightarrow p}=\left[\operatorname{det}\left[\left(\boldsymbol{\mu}+\frac{1}{2} \mathbf{K}^{*} \mathbf{K}+\frac{1}{2} \mathbf{I}_{B}\right)^{p}-\left(\boldsymbol{\mu}+\frac{1}{2} \mathbf{K}^{*} \mathbf{K}-\frac{1}{2} \mathbf{I}_{B}\right)^{p}\right]\right]^{-1 / p}
$$

и

$$
\check{H}(\Phi)=\operatorname{tr} g\left(\boldsymbol{\mu}+\frac{1}{2}\left(\mathbf{K}^{*} \mathbf{K}-\mathbf{I}_{B}\right)\right),
$$

где $g(x)=(x+1) \log (x+1)-x \log x$ и $\operatorname{tr}$ обозначает след операторов в $\mathbf{Z}$. В последнем случае мы использовали формулу для энтропии гауссовского состояния (48) (см. [40]):

$$
H(\rho)=\operatorname{tr} g\left(\boldsymbol{\alpha}-\frac{1}{2} \mathbf{I}\right)
$$

Теперь обратимся к классической пропускной способности канала $\Phi$. В бесконечномерном случае появляются два новых отличия по сравнению с ситуацией, рассмотренной в п. 2.4. Во-первых, понятие ансамбля должно быть обобщено на непрерывные семейства состояний. Назовем обобщенным ансамблем произвольную борелевскую вероятностную меру $\pi$ на $\mathfrak{S}\left(\mathscr{H}_{A}\right)$. Среднее состояние обобщенного ансамбля $\pi$ определяется как барицентр вероятностной меры:

$$
\bar{\rho}_{\pi}=\int_{\mathfrak{S}\left(\mathscr{H}_{A}\right)} \rho \pi(d \rho) .
$$

Обычные ансамбли соответствуют мерам с конечным носителем.

Во-вторых, приходится ввести ограничение на входе канала, чтобы избежать бесконечных пропускных способностей. Пусть $F$ - положительный самосопряженный оператор в $\mathscr{H}_{A}$, который обычно представляет собой энергию системы $A$. Мы рассматриваем состояния на входе с ограниченной энергией: $\operatorname{Tr} \rho F \leqslant E$, где $E$ - фиксированная положительная константа. Поскольку оператор $F$ обычно неограничен, требуется аккуратность при определении следа; мы полагаем $\operatorname{Tr} \rho F=\int_{0}^{\infty} \lambda d m_{\rho}(\lambda)$, где $m_{\rho}(\lambda)=\operatorname{Tr} \rho E(\lambda)$ и $E(\lambda)$ - спектральная функция самосопряженного оператора $F$. Тогда $\chi$-пропускная способность с ограничением дается следующим обобщением выражения (17):

$$
C_{\chi}(\Phi, F, E)=\sup _{\pi: \operatorname{Tr} \bar{\rho}_{\pi} F \leqslant E} \chi(\pi),
$$

где

$$
\chi(\pi)=H\left(\Phi\left[\bar{\rho}_{\pi}\right]\right)-\int_{\mathfrak{S}\left(\mathscr{H}_{A}\right)} H(\Phi[\rho]) \pi(d \rho) .
$$


Чтобы гарантировать корректность определения этого выражения, необходимы некоторые условия на канал $\Phi$ и на оператор ограничения $F$ (см. [35], [38; п. 11.5], [58]), которые, однако, всегда выполняются в гауссовском случае, рассматриваемом ниже.

Обозначим $F^{(n)}=F \otimes I \cdots \otimes I+\cdots+I \otimes \cdots \otimes I \otimes F$, тогда классическая пропускная способность с ограничением дается выражением

$$
C(\Phi, F, E)=\lim _{n \rightarrow \infty} \frac{1}{n} C_{\chi}\left(\Phi^{\otimes n}, F^{(n)}, n E\right) .
$$

Пусть теперь $\Phi$ - гауссовский калибровочно-ковариантный канал. Рассмотрим калибровочно-инвариантный осцилляторный оператор энергии

$$
F=\sum_{j, k=1}^{s_{A}} \epsilon_{j k} a_{j}^{*} a_{k}
$$

где $\boldsymbol{\epsilon}=\left[\epsilon_{j k}\right]$ - эрмитова положительно определенная матрица и $a_{j}=\left(q_{j}+\right.$ $\left.i p_{j}\right) / \sqrt{2}$ - оператор уничтожения для $j$-й моды. Для любого состояния $\rho$, удовлетворяющего условию $\operatorname{Tr} \rho F<\infty$, корректно определены первые моменты $\operatorname{Tr} \rho a_{j}$ и вторые моменты $\operatorname{Tr} \rho a_{j}^{*} a_{k}, \operatorname{Tr} \rho a_{j} a_{k}$. Для калибровочно-инвариантного состояния имеем $\operatorname{Tr} \rho a_{j}=0$ и $\operatorname{Tr} \rho a_{j} a_{k}=0$. Для гауссовского калибровочно-инвариантного состояния (48) имеем

$$
\boldsymbol{\alpha}-\frac{\mathbf{I}}{2}=\left[\operatorname{Tr} \bar{\rho}_{\pi} a_{j}^{*} a_{k}\right]_{j, k=1, \ldots, s}
$$

(см., например, [33]).

ПредлОЖЕНИЕ 17. Классическая пропускная способность с ограничением гауссовского калибровочно-ковариантного канала $\Phi$ равна

$$
\begin{gathered}
C(\Phi ; F, E)=C_{\chi}(\Phi ; F, E)=\max _{\boldsymbol{\nu}: \operatorname{tr} \boldsymbol{\nu} \epsilon \leqslant E} \operatorname{tr} g\left(\mathbf{K}^{*} \boldsymbol{\nu} \mathbf{K}+\boldsymbol{\mu}+\frac{1}{2}\left(\mathbf{K}^{*} \mathbf{K}-\mathbf{I}_{B}\right)\right) \\
-\operatorname{tr} g\left(\boldsymbol{\mu}+\frac{1}{2}\left(\mathbf{K}^{*} \mathbf{K}-\mathbf{I}_{B}\right)\right) .
\end{gathered}
$$

Оптимальный ансамбль $\pi$, на котором достигается супремум в (86), состоит из когерентных состояний $\rho_{\mathbf{z}}=D_{A}(\mathbf{z}) \rho_{0} D_{A}(\mathbf{z})^{*}, \mathbf{z} \in \mathbf{Z}_{A}$, с калибровочно-инвариантным гауссовским распределением вероятностей $Q_{\nu}\left(d^{2 s} z\right)$ на $\mathbf{Z}_{A}$, имеющим нулевое среднее и корреляиионную матрицу $\nu$, которая является решением максимизационной проблемы в (89).

ДокАЗАТЕЛЬство. Рассмотрим гауссовский ансамбль $\pi_{\boldsymbol{\nu}}$, состоящий из когерентных состояний $\rho_{\mathbf{z}}=D_{A}(\mathbf{z}) \rho_{0} D_{A}(\mathbf{z})^{*}, \mathbf{z} \in \mathbf{Z}_{A}$, с калибровочно-инвариантным гауссовским распределением вероятностей $Q_{\nu}\left(d^{2 s} z\right)$ на $\mathbf{Z}_{A}$, имеющим нулевое среднее и некоторую корреляционную матрицу $\boldsymbol{\nu}$. Распределение задается классической характеристической функцией

$$
\int_{\mathbf{Z}_{A}} \exp \left\{2 i \operatorname{Im} \mathbf{w}^{*} \mathbf{z}\right\} Q_{\boldsymbol{\nu}}\left(d^{2 s} w\right)=\exp \left\{-\mathbf{z}^{*} \boldsymbol{\nu} \mathbf{z}\right\} .
$$


Используя свойство ковариантности (34) гауссовского канала, получаем

$$
H\left(\Phi\left[\rho_{\mathbf{z}}\right]\right)=H\left(\Phi\left[D_{A}(\mathbf{z}) \rho_{0} D_{A}(\mathbf{z})^{*}\right]\right)=H\left(\Phi\left[\rho_{0}\right]\right)=\operatorname{tr} g\left(\boldsymbol{\mu}+\frac{1}{2}\left(\mathbf{K}^{*} \mathbf{K}-\mathbf{I}_{B}\right)\right),
$$

что не зависит от $\mathbf{z}$ и, следовательно, дает значение интегрального члена в (87). Интегрируя характеристические функции когерентных состояний, получаем

$$
\operatorname{Tr} \bar{\rho}_{\pi_{\nu}} D_{A}(\mathbf{z})=\exp \left\{-\mathbf{z}^{*}\left(\boldsymbol{\nu}+\frac{1}{2} \mathbf{I}_{A}\right) \mathbf{z}\right\} .
$$

Тогда

$$
\boldsymbol{\nu}=\left[\operatorname{Tr} \bar{\rho}_{\pi_{\nu}} a_{j}^{*} a_{k}\right]_{j, k=1, \ldots, s_{A}} \quad \text { и } \quad \operatorname{Tr} \bar{\rho}_{\pi_{\nu}} F=\sum_{j, k=1}^{s} \epsilon_{j k} \operatorname{Tr} \bar{\rho}_{\pi_{\nu}} a_{j}^{*} a_{k}=\operatorname{tr} \boldsymbol{\nu} \boldsymbol{\epsilon} .
$$

Состояние $\Phi\left[\bar{\rho}_{\pi_{\nu}}\right]$ является калибровочно-инвариантным гауссовским с корреляционной матрицей $\mathbf{K}^{*}\left(\boldsymbol{\nu}+\mathbf{I}_{A} / 2\right) \mathbf{K}+\boldsymbol{\mu}$, поэтому оно имеет энтропию

$$
\operatorname{tr} g\left(\mathbf{K}^{*} \boldsymbol{\nu} \mathbf{K}+\boldsymbol{\mu}+\frac{1}{2}\left(\mathbf{K}^{*} \mathbf{K}-\mathbf{I}_{B}\right)\right) .
$$

Таким образом, для гауссовского ансамбля $\pi_{\boldsymbol{\nu}}$

$$
\chi\left(\pi_{\boldsymbol{\nu}}\right)=\operatorname{tr} g\left(\mathbf{K}^{*} \boldsymbol{\nu} \mathbf{K}+\boldsymbol{\mu}+\frac{1}{2}\left(\mathbf{K}^{*} \mathbf{K}-\mathbf{I}_{B}\right)\right)-\operatorname{tr} g\left(\boldsymbol{\mu}+\frac{1}{2}\left(\mathbf{K}^{*} \mathbf{K}-\mathbf{I}_{B}\right)\right) .
$$

В итоге мы должны доказать, что

$$
C(\Phi ; F, E)=C_{\chi}(\Phi ; F, E)=\sup _{\boldsymbol{\nu}: \operatorname{tr} \boldsymbol{\nu} \leqslant \leqslant E} \chi\left(\pi_{\boldsymbol{\nu}}\right) .
$$

Обозначим $\mathscr{G}$ множество всех гауссовских калибровочно-инвариантных состояний в $\mathscr{H}_{A}$.

Лемма 18. Имеет место неравенство

$$
\max _{\rho^{(n)}: \operatorname{Tr} \rho^{(n)} F^{(n)} \leqslant n E} H\left(\Phi^{\otimes n}\left[\rho^{(n)}\right]\right) \leqslant n \max _{\rho: \rho \in \mathscr{G}, \operatorname{Tr} \rho F \leqslant E} H(\Phi[\rho]) .
$$

ДокАзАтельство. Докажем сначала, что

$$
\sup _{\rho^{(n)}: \operatorname{Tr} \rho^{(n)} F^{(n)} \leqslant n E} H\left(\Phi^{\otimes n}\left[\rho^{(n)}\right]\right) \leqslant n \sup _{\rho: \operatorname{Tr} \rho F \leqslant E} H(\Phi[\rho]) .
$$

В самом деле, обозначая $\rho_{j}$ частичное состояние $\rho^{(n)}$ в $j$-м тензорном множителе произведения $\mathscr{H}_{A}^{\otimes n}$ и полагая $\bar{\rho}=n^{-1} \sum_{j=1}^{n} \rho_{j}$, имеем

$$
H\left(\Phi^{\otimes n}\left[\rho^{(n)}\right]\right) \leqslant \sum_{j=1}^{n} H\left(\Phi\left[\rho_{j}\right]\right) \leqslant n H(\Phi[\bar{\rho}]),
$$


где в первом неравенстве мы использовали субаддитивность квантовой энтропии, а во втором - ее вогнутость. Более того, $\operatorname{Tr} \bar{\rho} F=n^{-1} \operatorname{Tr} \rho^{(n)} F^{(n)} \leqslant E$, откуда следует (93).

Используя калибровочную ковариантность канала $\Phi$, мы можем ограничить максимизацию в правой части (93) калибровочно-инвариантными состояниями. В самом деле, для данного состояния $\rho$, удовлетворяющего ограничению $\operatorname{Tr} \rho F \leqslant E$, его усреднение

$$
\rho_{\mathrm{av}}=\frac{1}{2 \pi} \int_{0}^{2 \pi} U_{\varphi} \rho U_{\varphi}^{*} d \varphi
$$

также удовлетворяет ограничению, тогда как $H(\Phi[\rho]) \leqslant H\left(\Phi\left[\rho_{\mathrm{av}}\right]\right)$ в силу вогнутости энтропии.

Наконец, используем принцип максимальной энтропии, согласно которому гауссовское состояние имеет максимальную энтропию среди всех состояний с фиксированными вторыми моментами (см., например, [38; лемма 12.25]). Это доказывает (92). Лемма доказана.

Теперь имеем

$$
\max _{\rho: \rho \in \mathscr{G}, \operatorname{Tr} \rho F \leqslant E} H(\Phi[\rho])=\max _{\boldsymbol{\nu}: \operatorname{tr} \boldsymbol{\nu} \in \leqslant E} \operatorname{tr} g\left(\mathbf{K}^{*} \boldsymbol{\nu} \mathbf{K}+\boldsymbol{\mu}+\frac{1}{2}\left(\mathbf{K}^{*} \mathbf{K}-\mathbf{I}_{B}\right)\right) .
$$

Пусть $\boldsymbol{\nu}$ - решение максимизационной проблемы в правой части (94). Чтобы доказать (89), заметим, что

$$
\begin{aligned}
n \chi\left(\pi_{\nu}\right) & \leqslant n C_{\chi}(\Phi, F, E) \leqslant C_{\chi}\left(\Phi^{\otimes n}, F^{(n)}, n E\right) \\
& \leqslant \max _{\rho^{(n)}: \operatorname{Tr} \rho^{(n)} F^{(n)} \leqslant n E} H\left(\Phi^{\otimes n}\left[\rho^{(n)}\right]\right)-\min _{\rho^{(n)}} H\left(\Phi^{\otimes n}\left[\rho^{(n)}\right]\right) .
\end{aligned}
$$

Используя лемму 18 и предложение 16, мы видим, что последнее выражение не превосходит

$$
n\left[\max _{\rho: \rho \in \mathscr{G}, \operatorname{Tr} \rho F \leqslant E} H(\Phi[\rho])-H\left(\Phi\left[\rho_{0}\right]\right)\right]=n \chi\left(\pi_{\nu}\right),
$$

где равенство следует из (94) и (90).

Таким образом, $C_{\chi}\left(\Phi^{\otimes n}, F^{(n)}, n E\right)=n C_{\chi}(\Phi, F, E)$, и поэтому классическая пропускная способность с ограничением (88) гауссовского калибровочно-ковариантного канала дается выражением (89). Предложение 17 доказано.

Аналогичные рассуждения применимы к гауссовскому калибровочно-ковариантному каналу (53), приводя к выражению (89) с заменой $\boldsymbol{\epsilon}$ на $\overline{\boldsymbol{\epsilon}}$. В самом деле, в этом случае состояние $\Phi\left[\bar{\rho}_{\pi_{\nu}}\right]$ является калибровочно-инвариантным гауссовским с характеристической функцией

$$
\begin{aligned}
\operatorname{Tr} \Phi\left[\bar{\rho}_{\pi_{\boldsymbol{\nu}}}\right] D(\mathbf{z}) & =\exp \left\{-(\overline{\mathbf{K} \mathbf{z}})^{*}\left(\boldsymbol{\nu}+\frac{1}{2} \mathbf{I}_{A}\right) \overline{\mathbf{K} \mathbf{z}}-\mathbf{z}^{*} \boldsymbol{\mu} \mathbf{z}\right\} \\
& =\exp \left\{-(\mathbf{K} \mathbf{z})^{*}\left(\overline{\boldsymbol{\nu}}+\frac{1}{2} \mathbf{I}_{A}\right) \mathbf{K z}-\mathbf{z}^{*} \boldsymbol{\mu} \mathbf{z}\right\}
\end{aligned}
$$


и корреляционной матрицей $\mathbf{K}^{*}\left(\overline{\boldsymbol{\nu}}+\mathbf{I}_{A} / 2\right) \mathbf{K}+\boldsymbol{\mu}$. С другой стороны, $\operatorname{tr} \boldsymbol{\nu} \boldsymbol{\epsilon}=\operatorname{tr} \overline{\boldsymbol{\nu}}$, так что, переопределяя $\overline{\boldsymbol{\nu}}$ как $\boldsymbol{\nu}$, мы и получаем утверждение.

Максимизация в (89) является конечномерной задачей оптимизации, которая является квантовым аналогом проблемы "water-filling" в классической теории информации (см., например, [15], [40]). Она решается аналитически в специальных случаях, что является предметом отдельного исследования.

3.6. Случай квантово-классического гауссовского канала. В этом пункте, следуя работе [23], мы даем альтернативное доказательство свойства мажоризации для глауберовских когерентных состояний, полученного в работе [52].

Рассмотрим аффинное отображение, которое переводит квантовые состояния $\rho \in \mathfrak{S}(\mathscr{H})$ в плотности распределения вероятностей на $\mathbf{Z}$ согласно формуле

$$
\rho \rightarrow p_{\rho}(\mathbf{z})=\operatorname{Tr} \rho D(\mathbf{z}) \rho_{0} D(\mathbf{z})^{*},
$$

где $D(\mathbf{z})$ - операторы смещения, $\rho_{0}$ - вакуумное состояние с квантовой характеристической функцией

$$
\phi_{0}(\mathbf{z}) \equiv \operatorname{Tr} \rho_{0} D(\mathbf{z})=\exp \left\{-\frac{1}{2}|\mathbf{z}|^{2}\right\} .
$$

Функция $p_{\rho}(\mathbf{z})$ ограничена единицей и является непрерывной плотностью распределения вероятностей, причем нормировка следует из разложения единичного оператора в $\mathscr{H}$ :

$$
\int_{\mathbf{Z}} D(\mathbf{z}) \rho_{0} D(\mathbf{z})^{*} \frac{d^{2 s} \mathbf{z}}{\pi^{s}}=I_{\mathscr{H}} .
$$

ПРЕДЛОЖЕНИЕ 19. Пусть $f$ - вогнутая функиия на $[0,1]$ u $f(0)=0$, тогда для произвольного состояния $\rho$

$$
\int_{\mathbf{Z}} f\left(p_{\rho}(\mathbf{z})\right) \frac{d^{2 s} \mathbf{z}}{\pi^{s}} \geqslant \int_{\mathbf{Z}} f\left(p_{\rho_{\mathbf{w}}}(\mathbf{z})\right) \frac{d^{2 s} \mathbf{z}}{\pi^{s}},
$$

где $\rho_{\mathbf{w}}-$ произвольное когерентное состояние.

ДокАзАТЕльство. Для любого $c>0$ рассмотрим канал $\Phi_{c}$, определенный соотношением

$$
\Phi_{c}[\rho]=\int \frac{d^{2 s} \mathbf{z}}{\pi^{s} c^{2 s}} \operatorname{Tr}\left[\rho D\left(c^{-1} \mathbf{z}\right) \rho_{0} D^{*}\left(c^{-1} \mathbf{z}\right)\right] \rho_{\mathbf{z}} .
$$

Отображение (97) является гауссовским калибровочно-ковариантным каналом таким, что

$$
\Phi_{c}^{*}[D(\mathbf{z})]=D(c \mathbf{z}) \exp \left\{-\frac{c^{2}+1}{2}|\mathbf{z}|^{2}\right\}
$$

(ср. [22; раздел 5]). Поэтому согласно теореме 14

$$
\operatorname{Tr} f\left(\Phi_{c}[\rho]\right) \geqslant \operatorname{Tr} f\left(\Phi_{c}\left[\rho_{\mathbf{w}}\right]\right)
$$

для всех состояний $\rho$ и любого когерентного состояния $\rho_{\mathbf{w}}$. Мы докажем предложение 19, переходя к пределу при $c \rightarrow \infty$. 
В доказательстве мы используем неравенства Березина-Либа [9]:

$$
\int_{\mathbf{Z}} f(\underline{p}(\mathbf{z})) \frac{d^{2 s} \mathbf{z}}{\pi^{s}} \leqslant \operatorname{Tr} f(\sigma) \leqslant \int_{\mathbf{Z}} f(\bar{p}(\mathbf{z})) \frac{d^{2 s} \mathbf{z}}{\pi^{s}},
$$

которые выполняются для любого квантового состояния, допускающего представление

$$
\sigma=\int_{\mathbf{Z}} \underline{p}(\mathbf{z}) \rho_{\mathbf{z}} \frac{d^{2 s} \mathbf{z}}{\pi^{s}}
$$

с плотностью распределения вероятностей $\underline{p}(\mathbf{z})$. В правой части $(99) \bar{p}(\mathbf{z})=$ $\operatorname{Tr} \sigma \rho_{\mathbf{z}}$. В неравенствах (99) следует предположить, что $f$ определена на $[0, \infty)$ (в самом деле, функция $\underline{p}(\mathbf{z})$ может быть неограниченной). Мы временно предположим это.

Полагая $\sigma=\Phi_{c}[\rho]$, из $(97)$ получаем

$$
\underline{p}(\mathbf{z})=\frac{1}{c^{2 s}} \operatorname{Tr} \rho D\left(c^{-1} \mathbf{z}\right) \rho_{0} D^{*}\left(c^{-1} \mathbf{z}\right)=\frac{1}{c^{2 s}} p_{\rho}\left(c^{-1} \mathbf{z}\right),
$$

тогда как

$$
\bar{p}(\mathbf{z})=\operatorname{Tr} \rho_{\mathbf{z}} \Phi_{c}[\rho]=\int_{\mathbf{z}} \underline{p}(\mathbf{w}) \operatorname{Tr} \rho_{\mathbf{z}} \rho_{\mathbf{w}} \frac{d^{2 s} \mathbf{w}}{\pi^{s}} .
$$

Используем известное соотношение (см., например, [48], [33])

$$
\operatorname{Tr} \rho_{\mathbf{z}} \rho_{\mathbf{w}}=\exp \left\{-|\mathbf{z}-\mathbf{w}|^{2}\right\}
$$

Вводя плотность гауссовского распределения вероятностей

$$
q_{c}(\mathbf{z})=\frac{c^{2 s}}{\pi^{s}} \exp \left\{-c^{2}|\mathbf{z}|^{2}\right\}
$$

которая стремится к $\delta$-функции при $c \rightarrow \infty$, и подставляя в $(100)$, имеем

$$
\begin{aligned}
\bar{p}(\mathbf{z}) & =\int d^{2 s} \mathbf{w} \underline{p}(\mathbf{w}) q_{1}(\mathbf{z}-\mathbf{w}) \\
& =\int d^{2 s} \mathbf{w}^{\prime} p_{\rho}\left(\mathbf{w}^{\prime}\right) q_{1}\left(\mathbf{z}-c \mathbf{w}^{\prime}\right) \\
& =\frac{1}{c^{2 s}} p_{\rho} * q_{c}\left(c^{-1} \mathbf{z}\right) .
\end{aligned}
$$

После замены переменных $c^{-1} \mathbf{z} \rightarrow \mathbf{z}$ неравенства (99) принимают вид

$$
\int_{\mathbf{Z}} f\left(c^{-2 s} p_{\rho}(\mathbf{z})\right) \frac{d^{2 s} \mathbf{z}}{\pi^{s}} \leqslant c^{-2 s} \operatorname{Tr} f\left(\Phi_{c}[\rho]\right) \leqslant \int_{\mathbb{C}^{s}} f\left(c^{-2 s} p_{\rho} * q_{c}(\mathbf{z})\right) \frac{d^{2 s} \mathbf{z}}{\pi^{s}} .
$$


Подставляя $\rho=\rho_{\mathbf{w}}$, получаем

$$
\int_{\mathbf{Z}} f\left(c^{-2 s} p_{\rho_{\mathbf{w}}}(\mathbf{z})\right) \frac{d^{2 s} \mathbf{z}}{\pi^{s}} \leqslant c^{-2 s} \operatorname{Tr} f\left(\Phi_{c}\left[\rho_{\mathbf{w}}\right]\right) \leqslant \int_{\mathbf{Z}} f\left(c^{-2 s} p_{\rho_{\mathbf{w}}} * q_{c}(\mathbf{z})\right) \frac{d^{2 s} \mathbf{z}}{\pi^{s}} .
$$

Комбинируя последние две формулы с неравенством (98), получаем

$$
\begin{aligned}
\int_{\mathbf{Z}} g & \left(p_{\rho}(\mathbf{z})\right) \frac{d^{2 s} \mathbf{z}}{\pi^{s}}-\int_{\mathbb{C}^{s}} g\left(p_{\rho_{\mathbf{w}}}(\mathbf{z})\right) \frac{d^{2 s} \mathbf{z}}{\pi^{s}} \\
& \geqslant \int_{\mathbf{Z}} g\left(p_{\rho}(\mathbf{z})\right) \frac{d^{2 s} \mathbf{z}}{\pi^{s}}-\int_{\mathbf{Z}} g\left(p_{\rho} * q_{c}(\mathbf{z})\right) \frac{d^{2 s} \mathbf{z}}{\pi^{s}},
\end{aligned}
$$

где введена функция $g(x)=f\left(c^{-2 s} x\right)$, которая также является вогнутой. Далее, произвольная вогнутая полигональная функция $g$ на $[0,1]$, удовлетворяющая условию $g(0)=0$, может быть получена таким образом из функции

$$
f(x)= \begin{cases}g\left(c^{2 s} x\right), & x \in\left[0, c^{-2 s}\right] \\ g(1)+g^{\prime}(1)\left(x-c^{-2 s}\right), & x \in\left[c^{-2 s}, \infty\right)\end{cases}
$$

поэтому (102) выполняется для всякой такой функции. Тогда правая часть неравенства (102) стремится к нулю при $c \rightarrow \infty$. В самом деле, для полигональной функции выполнено $|g(x)-g(y)| \leqslant \varkappa|x-y|$, и требуемая сходимость вытекает из сходимости $p_{\rho} * q_{c} \rightarrow p_{\rho}$ в $L_{1}$ : если $p(\mathbf{z})$ - ограниченная непрерывная плотность распределения вероятностей, то

$$
\lim _{c \rightarrow \infty} \int_{\mathbf{Z}}\left|p * q_{c}(\mathbf{z})-p(\mathbf{z})\right| d^{2 s} \mathbf{z}=0 .
$$

Таким образом, мы получили (96) для вогнутых полигональных функций $f$. Однако произвольную вогнутую функцию $f$ на $[0,1]$ можно приблизить монотонно неубывающей последовательностью вогнутых полигональных функций $f_{n}$. Применяя теорему Беппо Леви, получаем требуемое утверждение. Предложение доказано.

\section{4. Приложение}

Рассмотрим калибровочно-ковариантный канал $\Phi$, для которого матрицы $\mathbf{K}^{*} \mathbf{K}$ и $\boldsymbol{\mu}$ коммутируют (в частности, этому условию удовлетворяют экстремальные аттенюаторы и усилители). Такие каналы диагонализуемы в следующем смысле. Имеем

$$
\mathbf{K}=\mathbf{V}_{A} \mathbf{K}_{d} \mathbf{V}_{B}, \quad \boldsymbol{\mu}=\mathbf{V}_{B}^{*} \boldsymbol{\mu}_{d} \mathbf{V}_{B}
$$

где $\mathbf{V}_{A}, \mathbf{V}_{B}$ - унитарные матрицы, a $\mathbf{K}_{d}, \boldsymbol{\mu}_{d}$ - диагональные (прямоугольные) матрицы с неотрицательными числами на диагонали. Тогда $\mathbf{K}^{*} \mathbf{K}=$ $\mathbf{V}_{B}^{*} \mathbf{K}_{d}^{2} \mathbf{V}_{B}$ и

$$
\Phi[\rho]=U_{B} \Phi_{d}\left[U_{A} \rho U_{A}^{*}\right] U_{B}^{*},
$$


где $U_{A}, U_{B}$ - канонические унитарные ("метаплектические" [2]) преобразования, действующие в $\mathscr{H}_{A}, \mathscr{H}_{B}$ так, что

$$
U_{B}^{*} D_{B}(\mathbf{z}) U_{B}=D_{B}\left(\mathbf{V}_{B} \mathbf{z}\right), \quad U_{A}^{*} D_{A}(\mathbf{z}) U_{A}=D_{A}\left(\mathbf{V}_{A} \mathbf{z}\right)
$$

Чтобы описать действие “диагонального” канала $\Phi_{d}$ более детально, рассмотрим отдельно случаи $s_{A}=s_{B}, s_{A} \leqslant s_{B}$ и $s_{A}>s_{B}$.

В случае $s_{A}=s_{B}$ имеем

$$
\mathbf{K}_{d}=\operatorname{diag}\left[k_{j}\right]_{j=1, \ldots, s_{B}}, \quad \boldsymbol{\mu}_{d}=\operatorname{diag}\left[\mu_{j}\right]_{j=1, \ldots, s_{B}} .
$$

Тогда $\Phi_{d}=\bigotimes_{j=1}^{s_{B}} \Phi_{j}$, где, в понятных обозначениях,

$$
\Phi_{j}^{*}\left[D_{j}\left(z_{j}\right)\right]=D_{j}\left(k_{j} z_{j}\right) \exp \left\{-\mu_{j}\left|z_{j}\right|^{2}\right\}
$$

В случае $s_{A}<s_{B}$ имеем

$$
\mathbf{K}_{d}=\left[\begin{array}{c}
\operatorname{diag}\left[k_{j}\right]_{j=1, \ldots, s_{A}} \\
\mathbf{0}
\end{array}\right]
$$

где $\mathbf{0}$ - нулевой блок размера $\left(s_{B}-s_{A}\right) \times s_{A}$. Тогда

$$
\Phi_{d}[\rho]=\bigotimes_{j=1}^{s_{A}} \Phi_{j}[\rho] \otimes \rho_{0}^{\left[s_{A}+1, \ldots, s_{B}\right]},
$$

где для $j=1, \ldots, s_{A}$ одномодовые каналы $\Phi_{j}$ даются соотношением (104), а $\rho_{0}^{\left[s_{A}+1, \ldots, s_{B}\right]}-$ вакуумные состояния мод $s_{A}+1, \ldots, s_{B}$.

В случае $s_{A}>s_{B}$

$$
\mathbf{K}_{d}=\left[\operatorname{diag}\left[k_{j}\right]_{j=1, \ldots, s_{B}} \quad \mathbf{0}\right],
$$

где $\mathbf{0}$ обозначает нулевой блок размера $s_{B} \times\left(s_{A}-s_{B}\right)$, а

$$
\Phi_{d}[\rho]=\left(\bigotimes_{j=1}^{s_{A}} \Phi_{j}\right)\left[\operatorname{Tr}_{s_{B}+1, \ldots, s_{A}} \rho\right]
$$

где $\operatorname{Tr}_{s_{B}+1, \ldots, s_{A}}$ обозначает частичный след по последним $s_{A}-s_{B}$ модам оператора $\rho$.

Для экстремального калибровочно-контравариантного канала имеет место такая же редукция к диагональному виду, что и полученная выше для калибровочно-ковариантного канала.

Автор благодарен М. Е. Широкову (МИАН) за обсуждение и Дэвиду Дингу (Стэнфордский университет) за замеченные опечатки. 


\section{Список литературы}

[1] Г. Г. Амосов, А. С. Холево, Р. Ф. Вернер, "О гипотезе аддитивности в квантовой теории информации", Пробл. передачи информ., 36:4 (2000), 25-34; англ. пер.: G. G. Amosov, A. S. Holevo, R. F. Werner, "On the additivity conjecture in quantum information theory", Problems Inform. Transmission, 36:4 (2000), 305-313.

[2] Arvind, B. Dutta, N. Mukunda, R. Simon, "The real symplectic groups in quantum mechanics and optics", Pramana, 45:6 (1995), 471-497.

[3] G. Aubrun, S. Szarek, E. Werner, "Hastings's additivity counterexample via Dvoretzky's theorem", Comm. Math. Phys., 305:1 (2011), 85-97.

[4] K. M. R. Audenaert, "A note on the $p \rightarrow q$ norms of completely positive maps", Linear Algebra Appl., 430:4 (2009), 1436-1440.

[5] К.И. Бабенко, "Об одном неравенстве в теории интегралов Фурье", Изв. АН СССР. Сер. матем., 25:4 (1961), 531-542; англ. пер.: K. I. Babenko, "An inequality in the theory of Fourier integrals", Amer. Math. Soc. Transl. Ser. 2, 44, Amer. Math. Soc., Providence, RI, 1965, 115-128.

[6] W. Beckner, "Inequalities in Fourier analysis", Ann. of Math. (2), 102:1 (1975), 159-182.

[7] S. T. Belinschi, B. Collins, I. Nechita, Almost one bit violation for the additivity of the minimum output entropy, 2013 (v3 - 2014), 24 pp., arXiv: 1305.1567.

[8] C. H. Bennett, C. A. Fuchs, J. A. Smolin, "Entanglement-enhanced classical communication on a noisy quantum channel", Quantum communication, computing, and measurement, eds. O. Hirota, A. S. Holevo, C. M. Caves, Plenum, New York, 1997, 79-88.

[9] Ф.А. Березин, "Ковариантные и контравариантные символы операторов", Изв. АН СССР. Сер. матем., 36:5 (1972), 1134-1167; англ. пер.: F. A. Berezin, "Covariant and contravariant symbols of operators", Math. USSR-Izv., 6:5 (1972), $1117-1151$.

[10] F. G. S. L. Brandão, M. Horodecki, "On Hastings' counterexamples to the minimum output entropy additivity conjecture", Open Syst. Inf. Dyn., 17:1 (2010), 31-52.

[11] E. Carlen, "Trace inequalities and quantum entropy: an introductory course", Entropy and the quantum, Contemp. Math., 529, Amer. Math. Soc., Providence, RI, 2010, 73-140.

[12] E. A. Carlen, E. H. Lieb, "A Minkowski type trace inequality and strong subadditivity of quantum entropy", Differential operators and spectral theory, Amer. Math. Soc. Transl. Ser. 2, 189, Amer. Math. Soc., Providence, RI, 1999, 59-68.

[13] F. Caruso, V. Giovannetti, A. S. Holevo, "One-mode bosonic Gaussian channels: a full weak-degradability classification", New J. Phys., 8 (2006), 310, 18 pp.

[14] F. Caruso, J. Eisert, V. Giovannetti, A.S. Holevo, "Multi-mode bosonic Gaussian channels", New J. Phys., 10 (2008), 083030, 33 pp.

[15] T. M. Cover, J.A. Thomas, Elements of information theory, Wiley Ser. Telecom., John Wiley \& Sons, Inc., New York, 1991, xxiv+542 pp.

[16] E. B. Davies, Quantum theory of open systems, Academic Press, London-New York, 1976, x+171 pp.

[17] B. Demoen, P. Vanheuverzwijn, A. Verbeure, "Completely positive quasi-free maps on the CCR-algebra", Rep. Math. Phys., 15:1 (1979), 27-39.

[18] M. Fukuda, C. King, D. K. Moser, "Comments on Hastings' additivity counterexamples", Comm. Math. Phys., 296:1 (2010), 111-143.

[19] M. Fukuda, M. M. Wolf, "Simplifying additivity problems using direct sum constructions", J. Math. Phys., 48:7 (2007), 072101, 7 pp. 
[20] R. García-Patrón, C. Navarrete-Benlloch, S. Lloyd, J. H. Shapiro, N. J. Cerf, "Majorization theory approach to the Gaussian channel minimum entropy conjecture", Phys. Rev. Lett., 108:11 (2012), 110505, 5 pp.

[21] V. Giovannetti, S. Guha, S. Lloyd, L. Maccone, J. H. Shapiro, H. P. Yuen, "Classical capacity of the lossy bosonic channel: the exact solution", Phys. Rev. Lett., 92:2 (2004), 027902, 4 pp.

[22] V. Giovannetti, A. S. Holevo, R. Garcia-Patron, "A solution of Gaussian optimizer conjecture for quantum channels", Comm. Math. Phys., 334:3 (2015), 1553-1571.

[23] В. Джованнетти, А. С. Холево, А. Мари, "Мажоризация и аддитивность для многомодовых бозонных гауссовских каналов", ТМФ, 182:2 (2015), 338-349; англ. пер.: V. Giovannetti, A. S. Holevo, A. Mari, Theoret. and Math. Phys., 182:2 (2015), 284-293.

[24] V. Giovannetti, S. Lloyd, "Additivity properties of a Gaussian channel", Phys. Rev. A, 69:6 (2004), 062307, 9 pp.

[25] A. Grudka, M. Horodecki, Ł. Pankowski, Constructive counterexamples to additivity of minimum output Rényi entropy of quantum channels for all $p>2,2009$ (v2 2010), 4 pp., arXiv: 0911.2515.

[26] M. B. Hastings, "Superadditivity of communication capacity using entangled inputs", Nature Physics, 5:4 (2009), 255-257.

[27] M. Hayashi, H. Imai, K. Matsumoto, M.-B. Ruskai, T. Shimono, "Qubit channels which require four inputs to achieve capacity: implications for additivity conjectures", Quantum Inf. Comput., 5:1 (2005), 13-31.

[28] P. Hayden, The maximal p-norm multiplicativity conjecture is false, 2007, 12 pp., arXiv: 0707.3291.

[29] P. Hayden, A. Winter, "Counterexamples to the maximal p-norm multiplicativity conjecture for all $p>1$ ", Comm. Math. Phys., 284:1 (2008), 263-280.

[30] T. Heinosaari, A. S. Holevo, M. M. Wolf, "The semigroup structure of Gaussian channels", Quantum Inf. Comput., 10:7-8 (2010), 619-635.

[31] T. Hiroshima, "Additivity and multiplicativity properties of some Gaussian channels for Gaussian inputs", Phys. Rev. A, 73:1 (2006), 012330, 9 pp.

[32] A. S. Holevo, "The capacity of the quantum channel with general signal states", IEEE Trans. Inform. Theory, 44:1 (1998), 269-273.

[33] А.С. Холево, Вероятностные и статистические аспекты квантовой теории, 2-е изд., доп. и испр., Ин-т компьют. исслед., М.-Ижевск, 2003, 404 с.; англ. пер.: A.S. Holevo, Probabilistic and statistical aspects of quantum theory, 2nd ed., Quaderni. Monographs, 1, Edizioni della Normale, Pisa, 2011, xvi+323 pp.

[34] А. С. Холево, Статистическая структура квантовой теории, Ин-т компьют. исслед., М.-Ижевск, 2003, 191 с.; пер. с англ.: А. S. Holevo, Statistical structure of quantum theory, Lect. Notes Phys. Monogr., 67, Springer-Verlag, Berlin, 2001, $\mathrm{x}+159 \mathrm{pp}$.

[35] А. С. Холево, "Классические пропускные способности квантового канала с ограничением на входе", Теория вероятн. и ее примен., 48:2 (2003), 359-374; англ. пер.: А.S. Holevo, "Entanglement-assisted capacities of constrained quantum channels", Theory Probab. Appl., 48:2 (2004), 243-255.

[36] А. С. Холево, "Комплементарные каналы и проблема аддитивности", Теория вероятн. и ее примен., 51:1 (2006), 133-143; англ. пер.: А. S. Holevo, "Complementary channels and the additivity problem", Theory Probab. Appl., 51:1 (2007), 92-100.

[37] А. С. Холево, "Мультипликативность $p$-норм вполне положительных отображений и проблема аддитивности в квантовой теории информации", УМН, 61:2(368) (2006), 113-152; англ. пер.: A. S. Holevo, "Multiplicativity of $p$-norms of completely positive maps and the additivity problem in quantum information theory", Russian Math. Surveys, 61:2 (2006), 301-339. 
[38] А.С. Холево, Квантовые системы, каналь, информацил, МЦНМО, М., 2010, 327 с.; англ. пер.: A.S. Holevo, Quantum systems, channels, information. A mathematical introduction, de Gruyter Stud. Math. Phys., 16, de Gruyter, Berlin, 2012, xiv +349 pp.

[39] А.С. Холево, "Об экстремальных бозонных линейных каналах", ТМФ, 174:2 (2013), 331-341; англ. пер.: А.S. Holevo, "Extreme bosonic linear channels", Theoret. and Math. Phys., 174:2 (2013), 288-297.

[40] A. S. Holevo, M. Sohma, O. Hirota, "Capacity of quantum Gaussian channels", Phys. Rev. A, 59:3 (1999), 1820-1828.

[41] A.S. Holevo, M. Sohma, O. Hirota, "Error exponents for quantum channels with constrained inputs", Rep. Math. Phys., 46:3 (2000), 343-358.

[42] A.S. Holevo, R.F. Werner, "Evaluating capacities of bosonic Gaussian channels", Phys. Rev. A, 63:3 (2001), 032312, 14 pp.

[43] M. Horodecki, P. W. Shor, M. B. Ruskai, "Entanglement breaking channels", Rev. Math. Phys., 15:6 (2003), 629-641.

[44] C. King, "Maximal p-norms of entanglement breaking channels", Quantum Inf. Comput., 3:2 (2003), 186-190; 2002, 7 pp., arXiv: quant-ph/0212057.

[45] C. King, "Additivity for unital qubit channels", J. Math. Phys., 43:10 (2002), 4641-4653.

[46] C. King, "The capacity of the quantum depolarizing channel", IEEE Trans. Inform. Theory, 49:1 (2003), 221-229.

[47] C. King, K. Matsumoto, M. Nathanson, M. B. Ruskai, "Properties of conjugate channels with applications to additivity and multiplicativity", Markov Process. Related Fields, 13:2 (2007), 391-423.

[48] J. R. Klauder, E. C. G. Sudarshan, Fundamentals of quantum optics, W. A. Benjamin, Inc., New York-Amsterdam, 1968, xi+279 pp.

[49] А.И. Кострикин, Ю.И. Манин, Линейная алгебра и геометрия, 2-е изд., Наука, М., 1986, 304 с.; англ. пер.: А. I. Kostrikin, Yu.I. Manin, Linear algebra and geometry, Algebra, 1, Gordon and Breach Science Publishers, New York, 1989, $\mathrm{x}+309 \mathrm{pp}$.

[50] E.H. Lieb, "Proof of an entropy conjecture of Wehrl", Comm. Math. Phys., 62:1 (1978), 35-41.

[51] E. H. Lieb, "Gaussian kernels have only Gaussian maximizers", Invent. Math., 102:1 (1990), 179-208.

[52] E. H. Lieb, J. P. Solovej, "Proof of an entropy conjecture for Bloch coherent spin states and its generalizations", Acta Math., 212:2 (2014), 379-398.

[53] A. Mari, V. Giovannetti, A. S. Holevo, "Quantum state majorization at the output of bosonic Gaussian channels", Nature Communications, 5 (2014), 3826.

[54] V. Paulsen, Completely bounded maps and operator algebras, Cambridge Stud. Adv. Math., 78, Cambridge Univ. Press, Cambridge, 2002, xii+300 pp.

[55] М. Рид, Б. Саймон, Методы современной математической физики, т. 1: Функционалъный анализ, Мир, М., 1977, 357 с.; пер. с англ.: M. Reed, B. Simon, Methods of modern mathematical physics, v. I: Functional analysis, Academic Press, New York-London, 1972, xvii+325 pp.

[56] A. Serafini, J. Eisert, M. M. Wolf, "Multiplicativity of maximal output purities of Gaussian channels under Gaussian inputs", Phys. Rev. A, 71:1 (2005), 012320, 9 pp.

[57] М. Е. Широков, "О супераддитивности выпуклого замыкания выходной энтропии квантового канала", УМH, 61:6(372) (2006), 191-192; англ. пер.: M. E. Shirokov, "Superadditivity of the convex closure of the output entropy of a quantum channel", Russian Math. Surveys, 61:6 (2006), 1186-1188. 
[58] М.Е. Широков, “О свойствах квантовых каналов, связанных с классической пропускной способностью”, Теория вероятн. и ее примен., 52:2 (2007), 301-335; англ. пер.: М. E. Shirokov, "On properties of quantum channels related to their classical capacity", Theory Probab. Appl., 52:2 (2008), 250-276.

[59] P. W. Shor, "Additivity of the classical capacity of entanglement-breaking quantum channels", J. Math. Phys., 43:9 (2002), 4334-4340.

[60] P.W. Shor, "Equivalence of additivity questions in quantum information theory", Comm. Math. Phys., 246:3 (2004), 453-472.

[61] W. F. Stinespring, "Positive functions on $C^{*}$-algebras", Proc. Amer. Math. Soc., 6:2 (1955), 211-216.

[62] K. Temme, F. Pastawski, M. J. Kastoryano, "Hypercontractivity of quasi-free quantum semigroups", J. Phys. A, 47:40 (2014), 405303, 27 pp.; 2014, 26 pp., arXiv: 1403.5224 .

[63] A. Wehrl, "General properties of entropy", Rev. Modern Phys., 50:2 (1978), 221-260.

[64] R.F. Werner, A. S. Holevo, "Counterexample to an additivity conjecture for output purity of quantum channels", J. Math. Phys., 43:9 (2002), 4353-4357.

[65] A. Winter, "The maximum output $p$-norm of quantum channels is not multiplicative for any $p>2$ ", 2007 (v3 - 2008), 4 pp., arXiv: 0707.0402.

\section{Александр Семенович Холево}

Поступила в редакцию

(Alexander S. Holevo)

11.01 .2015

Математический институт им. В. А. Стеклова РАН

E-mail: holevo@mi.ras.ru 